\title{
RESEARCH
}

Open Access

\section{Single-colony sequencing reveals microbe- by-microbiome phylosymbiosis between the cyanobacterium Microcystis and its associated bacteria}

Olga M. Pérez-Carrascal ${ }^{1 *+}$, Nicolas Tromas ${ }^{1{ }^{*+}}$, Yves Terrat $^{1}$, Elisa Moreno ${ }^{1}$, Alessandra Giani², Laisa Corrêa Braga Marques ${ }^{2}$, Nathalie Fortin ${ }^{3}$ and B. Jesse Shapiro ${ }^{1,4,5^{*}}$

\begin{abstract}
Background: Cyanobacteria from the genus Microcystis can form large mucilaginous colonies with attached heterotrophic bacteria - their microbiome. However, the nature of the relationship between Microcystis and its microbiome remains unclear. Is it a long-term, evolutionarily stable association? Which partners benefit? Here we report the genomic diversity of 109 individual Microcystis colonies_including cyanobacteria and associated bacterial genomes-isolated in situ and without culture from Lake Champlain, Canada and Pampulha Reservoir, Brazil.

Results: We identified 14 distinct Microcystis genotypes from Canada, of which only two have been previously reported, and four genotypes specific to Brazil. Microcystis genetic diversity was much greater between than within colonies, consistent with colony growth by clonal expansion rather than aggregation of Microcystis cells. We also identified 72 bacterial species in the microbiome. Each Microcystis genotype had a distinct microbiome composition, and more closely related genotypes had more similar microbiomes. This pattern of phylosymbiosis could be explained by co-phylogeny in only two out of the nine most prevalent associated bacterial genera, Roseomonas and Rhodobacter. These phylogenetically associated genera could enrich the metabolic repertoire of Microcystis, for example by encoding the biosynthesis of complementary carotenoid molecules. In contrast, other colony-associated bacteria showed weaker signals of co-phylogeny, but stronger evidence of horizontal gene transfer with Microcystis. These observations suggest that acquired genes are more likely to be retained in both partners (Microcystis and members of its microbiome) when they are loosely associated, whereas one gene copy is sufficient when the association is physically tight and evolutionarily long-lasting.
\end{abstract}

\footnotetext{
*Correspondence: olga.maria.perez-carrascal@umontreal.ca;

nicolas.tromas@umontreal.ca; jesse.shapiro@mcgill.ca

${ }^{\dagger}$ Olga M. Pérez-Carrascal and Nicolas Tromas contributed equally to this work.

'Département de Sciences Biologiques, Université de Montréal, Montréal, Québec, Canada

Full list of author information is available at the end of the article
}

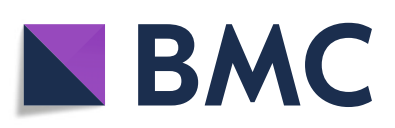

(- The Author(s). 2021 Open Access This article is licensed under a Creative Commons Attribution 4.0 International License, which permits use, sharing, adaptation, distribution and reproduction in any medium or format, as long as you give appropriate credit to the original author(s) and the source, provide a link to the Creative Commons licence, and indicate if changes were made. The images or other third party material in this article are included in the article's Creative Commons licence, unless indicated otherwise in a credit line to the material. If material is not included in the article's Creative Commons licence and your intended use is not permitted by statutory regulation or exceeds the permitted use, you will need to obtain permission directly from the copyright holder. To view a copy of this licence, visit http://creativecommons.org/licenses/by/4.0/. The Creative Commons Public Domain Dedication waiver (http://creativecommons.org/publicdomain/zero/1.0/) applies to the data made available in this article, unless otherwise stated in a credit line to the data. 
Conclusions: We have introduced a method for culture-free isolation of single colonies from nature followed by metagenomic sequencing, which could be applied to other types of microbes. Together, our results expand the known genetic diversity of both Microcystis and its microbiome in natural settings, and support their long-term, specific, and potentially beneficial associations.

Keywords: Microcystis, Cyanobacteria, Phylosymbiosis, Co-phylogeny, Microbiome, Phycosphere

\section{Background}

Cyanobacteria occur naturally in aquatic ecosystems, often multiplying into harmful blooms and producing a diversity of toxins, which can cause severe human illness [1]. Many cyanobacteria and eukaryotic algae grow in mucilaginous colonies surrounded by a zone, called the phycosphere, rich in cell exudates, where metabolites are exchanged between numerous microorganisms [2, 3]. In this microhabitat, the interactions between cyanobacteria and associated bacteria $(\mathrm{AB})$ might include mutualism (with all partners benefitting), competition (with all partners competing for resources), antagonism (inhibiting one of the partners), commensalism (with one partner benefitting) and parasitism (with one partner benefitting at the expense of the other) [3-5]. However, the drivers shaping these associations are largely unknown. In some cases, $A B$ may enhance algal or cyanobacterial growth $[6,7]$, aiding in phosphorus acquisition in Trichodesmium for example $[8,9]$. Understanding the contributions of $\mathrm{AB}$ to cyanobacterial growth and toxin production has implications for our ability to predict and control harmful blooms.

Microcystis is a globally distributed, often toxigenic bloom-forming freshwater cyanobacterium, which forms macroscopic mucilaginous colonies. These colonies offer a nutrient-rich habitat for other bacteria, while also providing physical protection against grazers [10-12]. The Microcystis colony microbiome is distinct from the surrounding lake bacterial community, enriched in Proteobacteria and depleted in Actinobacteria [13, 14]. The microbiome composition has been associated with temperature, seasonality, biogeography, Microcystis morphology and density [13, 15-17]. Lab experiments show the potential for $\mathrm{AB}$ to influence Microcystis growth and colony formation [18-21]. Yet it remains unclear whether such interactions are relevant in natural settings, and if they are the product of long-term associations over evolutionary time. Microcystis interacts with eukaryotes through competition with algae and predation by zooplankton [22], but eukaryotes are not known to be physically associated with Microcystis colonies. In contrast, sequencing and microscopy have revealed physical associations between Microcystis and smaller bacterial cells [23]. The in situ isolation of Microcystis from lakes offers an opportunity to further elucidate the associations between Microcystis and specific members of its microbiome.

Phylosymbiosis, a pattern in which microbiome composition mirrors the host phylogeny, provides a useful concept for the study of host-microbiome associations and interactions [24]. Phylosymbiosis could arise from some combination of (1) vertical transmission of the microbiome from parent to offspring, resulting in co-speciation and shared phylogenetic patterns (co-phylogeny), (2) horizontal transmission of the microbiome, but with strong matching between hosts and microbiomes at each generation, and (3) coevolution, in which hosts and microbiomes mutually impose selective pressures and adapt to each other. Distinguishing the relative importance of these three possibilities can be challenging, but in all cases, the associations between hosts and microbiomes must be significantly non-random in order for phylosymbiosis to be supported. Phylosymbiosis is typically studied between plant or animal hosts and their microbiomes [25-27] but Microcystis could also be considered a host, since it constructs the mucilage environmentalthough it is unclear to what extent it selects its $A B$ or vice versa. Microcystis colonies are more open to the outside environment compared with mammalian guts, for example. Consequently, they might behave more like coral mucus [27] or other animal surfaces which seem to show weaker phylosymbiosis than guts [28]. The enclosed nature of animal guts reduces dispersal of microbiomes and favours vertical transmission, potentially leading to co-phylogeny without the need to invoke co-evolution [29]. In contrast, metagenomic sequencing suggests Microcystis and its microbiome are globally distributed [16], making it unlikely that phylosymbiosis could arise due to common biogeography of Microcystis and its microbiome. On the other hand, Microcystis may be geographically structured on shorter evolutionary time scales, due to local adaptation or clonal expansions, and Microcystis genotypes might have distinct phenotypic characteristics that could select for distinct microbiomes [30, 31]. Phylosymbiosis studies to date are biased toward the gut relative to external host compartments [24]. Microcystis colonies thus provide an alternative model of a more external microbiome in which to study "microbe-by-microbiome" phylosymbiosis. 
Previous studies of the Microcystis microbiome have used either culture-independent metagenomics from lakes, a bulk biomass collection method which cannot resolve fine-scale spatial interaction within colonies (e.g., [16]), or culture-based studies of Microcystis isolates, which have found host-microbiome divergence according to phosphorous gradients and taxonomy [32], but may not be representative of the natural diversity of Microcystis or $\mathrm{AB}$ as they occur in nature. To combine the strengths of both these approaches, we developed a simple method for isolating individual Microcystis colonies directly from lakes, followed by DNA extraction and sequencing without a culture step [31]. Here we applied this method to 109 individual colonies from Lake Champlain, Canada and Pampulha Reservoir, Brazil, yielding 109 Microcystis genomes and 391 AB genomes. This genomic dataset allowed us (i) to quantify the Microcystis genotype diversity in lakes over time; (ii) to identify associations between Microcystis genotypes and microbiome composition and assess the evidence for phylosymbiosis; and finally, (iii) to test for possible horizontal gene transfer (HGT) between Microcystis and members of its microbiome.

Our findings reveal an expanded Microcystis genotypic diversity, and a Microcystis colony microbiome shaped by the host genotype, resulting in a significant signature of phylosymbiosis. We inferred co-speciation of Microcystis with two of the most prevalent genera in its microbiome (Rhodobacter and Roseomonas) suggesting evolutionarily stable associations. We also inferred HGT events among Microcystis and its microbiome, mainly involving lower-fidelity partners than Rhodobacter and Roseomonas. Overall, our results suggest ecologically and evolutionarily stable associations between Microcystis and members of its microbiome.

\section{Results}

\section{Genotypic diversity of Microcystis colonies in Lake} Champlain and Pampulha Reservoir

To study the relationship between Microcystis and its $\mathrm{AB}$ in natural settings, we sequenced 109 individual Microcystis colonies from 16 lake samples (82 colonies from Lake Champlain, Quebec, Canada and 27 from Pampulha Reservoir, Minas Gerais, Brazil; Supplementary Table 1). Microcystis genomes were assembled and binned separately from AB genomes (Methods), which we will describe below. Microcystis genomes recovered from the colonies have more than 90\% completeness and less than $10 \%$ redundance based on a set of 139 single-copy core genes [33], except for M04BS1, which has $82.7 \%$ completeness (Supplementary Table 1), with an average genome size of $4.76 \mathrm{Mb}$. Consistent with our previous study of Microcystis isolate genomes [31], nearly all the 109 Microcystis genomes and the 122 reference genomes (average genome size of $4.85 \mathrm{Mb}$ ) share $\geq 95 \%$ average nucleotide identity (ANI), except for 7 out of 26,565 genome pairs which had ANI between 94.4 and $94.5 \%$. The $95 \%$ ANI threshold is typically used to define bacterial species, but we previously found significant phylogenetic substructure above 95\%, coherent with multiple species or ecotypes within Microcystis [31]. In agreement with such fine genetic structure within our sampled colonies, we identified 18 monophyletic, closely related genotypes of Microcystis (i.e., $\geq$ 99.9\% ANI and phylogenetic pairwise distances lower than 0.0005; Supplementary Table 2 and Supplementary Fig. 1). These genotypes (highlighted clades in Fig. 1) are nested within the phylogeny of 122 isolate genomes previously sampled from North America, Brazil, and worldwide. However, only two genotypes (G05 and G10) have been reported in culture previously, possibly due to our fine-grained definition of genotypes ( $\geq 99 \%$ ANI) combined with undersampling of natural diversity in culture collections [34]. Consistent with previously observed biogeographic patterns between North and South America [31], we found 14 genotypes unique to Lake Champlain, and four unique to Pampulha, with no genotypes found in both locations.

Two features of interest that might distinguish Microcystis clades are toxigenicity (the presence of the mcy operon encoding microcystin biosynthesis [35]) and adaptation to low nutrient conditions. Microcystis is generally thought to be adapted to high nutrient conditions, since it often blooms in eutrophic waters such as Champlain and Pampulha (Supplementary Table 3). However, a recent sampling of Michigan lakes identified Microcystis isolates adapted to low-phosphorus (lowphosphorus genotypes, LG), which occur in both highand low-phosphorus lakes [32]. Genotypes G07, G08, G09 and G10 from Lake Champlain are nested within the LG clade with high bootstrap support (Fig. 1), indicating that low-phosphorus-adapted genotypes also occur in high-phosphorus lakes. Contrary to the single LG clade, high-phosphorus genotypes (HG) are broadly distributed across the phylogenetic tree and are recovered from multiple geographic locations. We observed that most of the genomes within the LG clade (66 out of 67) encode the mcy gene cluster (Fig. 1). In contrast, $m c y$ was more unevenly distributed across HG genomes, possibly due to multiple gene gain/loss events. However, closely related genotypes tend to have identical $m c y$ gene profiles, suggesting that putative gain/loss events occurred mainly in deep internal branches of the phylogeny.

Lower Microcystis diversity within than between colonies of the same genotype suggests clonal colony formation A previous study of Michigan lakes supported clonal colony formation (by cell division) in isolates from high- 


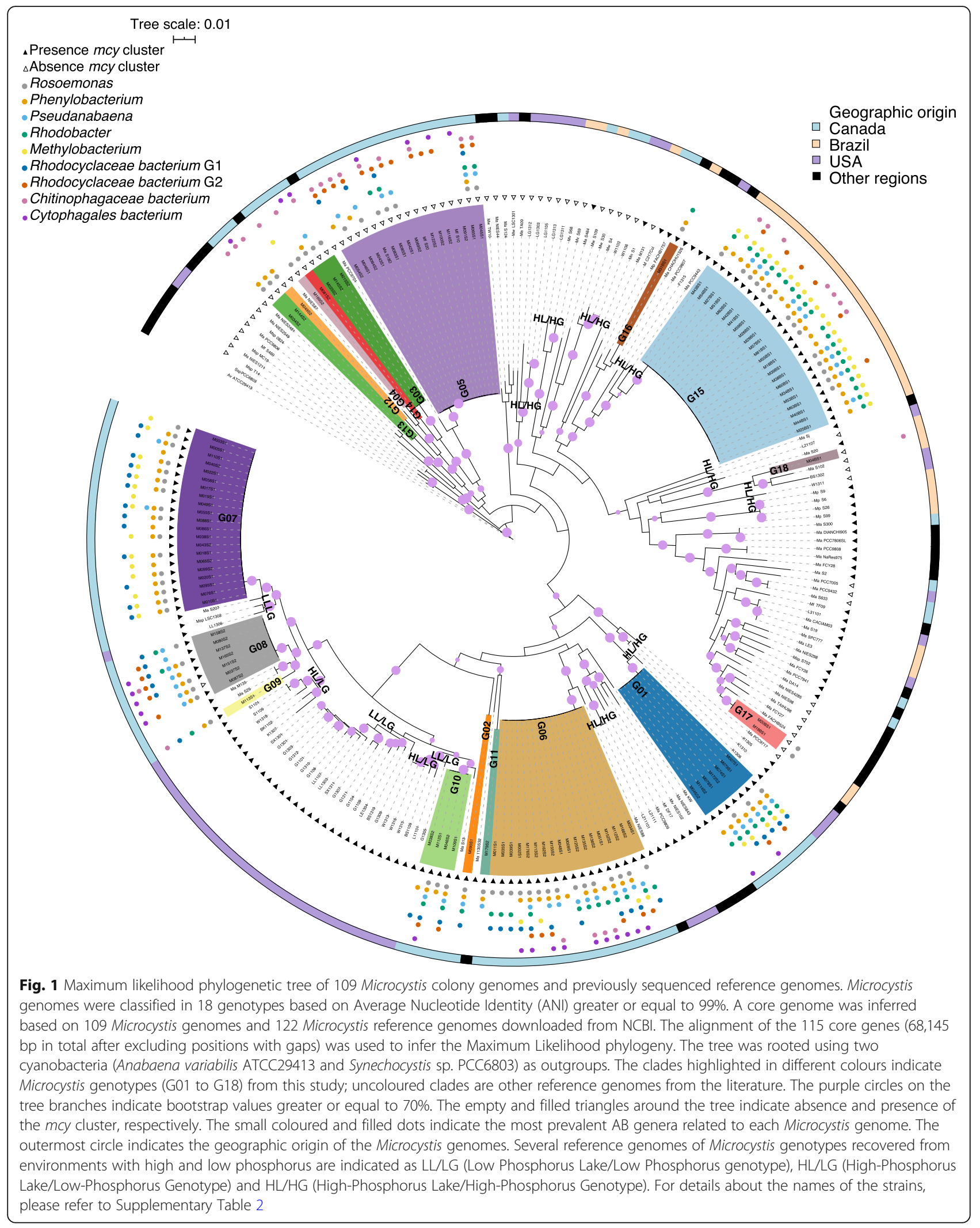


phosphorus lakes, but suggested a preponderance of nonclonal colonies (by agglomeration of distantly related cell) in low-phosphorus lakes [32]. To further explore whether Microcystis colonies emerge from a single clonal cell or from an aggregation of either genetically diverse, we compared genetic diversity within and between colonies of the same genotype. Note that Microcystis genotypes are defined by ANI values greater than $99.9 \%$ and phylogenetic pairwise distances lower than 0.0005 , and are thus not entirely isogenic. Within a colony, the number of single-nucleotide variants (SNVs) was significantly lower (mean of 3 and median of 2 SNVs) than between colonies (mean of 25 and median of 9 SNVs) of the same genotype (two-tailed Wilcoxon Rank Sum Test, $P<0.05$; twelve outliers with more than 300 variants between colonies were excluded, making the test conservative) (Fig. 2 and Supplementary Table 4). These outliers were found in colonies within the genotypes G05, G06, G08 and G13. To put these results in context, we compared isolate genomes from 19 laboratory cultures of Microcystis sequenced in a previous study [31]. Microcystis accumulated an average of $5 \mathrm{SNVs}$ after $\sim 6$ years of culture, with slightly more variation than observed within a colony but still $\sim 5$ times less than observed between

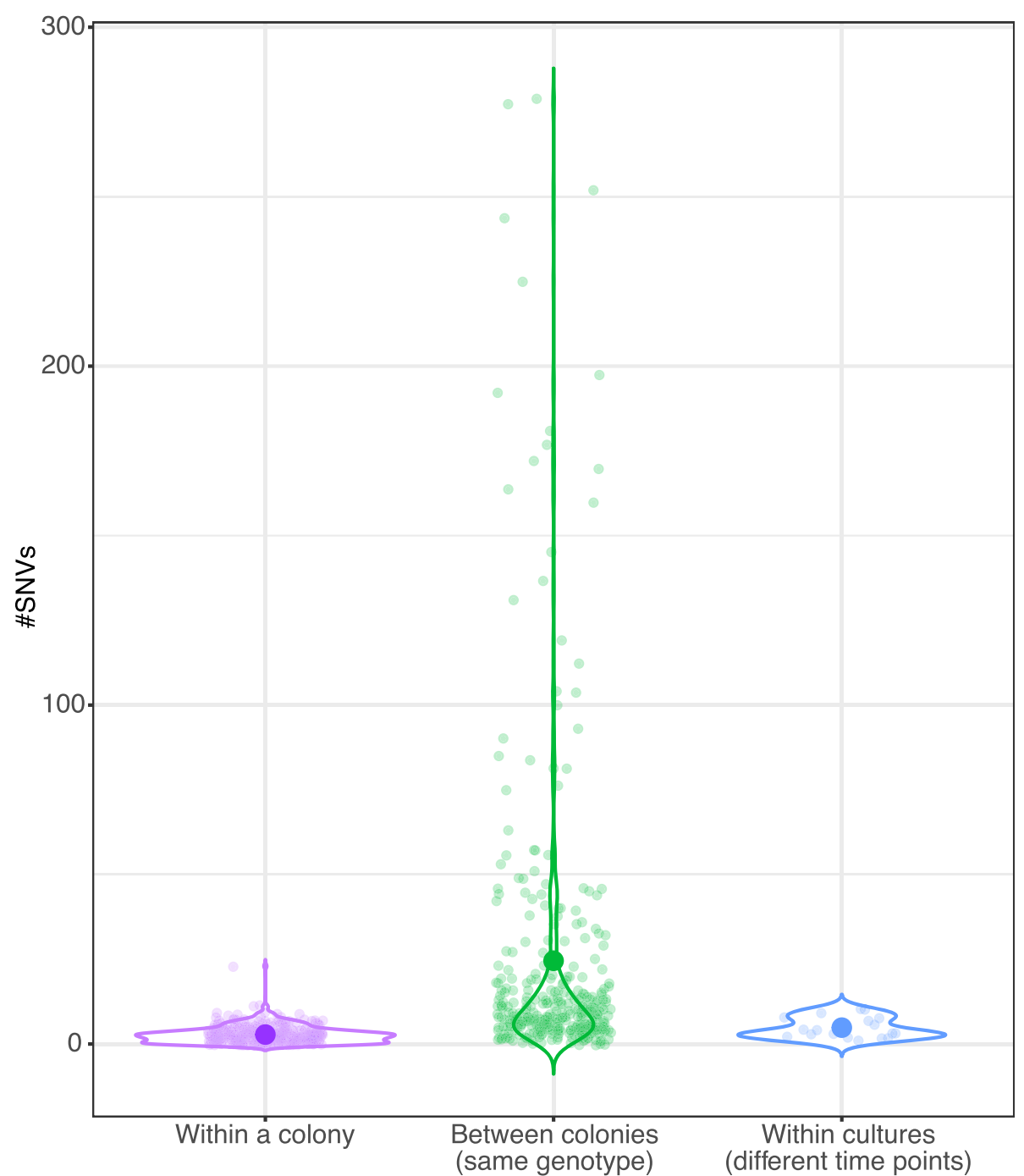

Fig. 2 Greater genetic diversity between than within Microcystis colonies. The number of single-nucleotide variants (SNVs) within and between Microcystis colonies of the same genotype are shown, compared with SNVs that accumulated over $\sim 6$ years of Microcystis culture in the laboratory (Methods). Large points show mean values. The data points represent the SNVs in a Microcystis genome within a colony (purple dots), SNVs between Microcystis genomes from the same genotypic clade (green dots), and SNVs between Microcystis genomes from the same Microcystis culture at two different time points (blue dots). Within colonies, the comparison was done by mapping the reads from a colony against its corresponding assembled Microcystis genome. Nucleotide sites that differed between some fraction of the reads and the assembled reference genome were considered as SNVs within a colony (the lowest allele frequency found within a colony was of $0.14 \%$ ) 
colonies of the same genotype (two-tailed Wilcoxon Rank Sum Test, $P<0.05)$. Overall, these results are consistent with clonal expansion of Microcystis genomes within a colony-at least under the sampled environmental conditions in Lake Champlain and Pampulha Reservoir.

\section{Evidence for phylosymbiosis between Microcystis and its microbiome}

Having characterized the genetic diversity of Microcystis, we turned our attention to the colony-associated bacteria (AB). We recovered a total of 391 non-Microcystis metagenome-assembled genomes (MAGs, with completeness $\geq 70$ and contamination < 10\%) from the 109 colonies (Supplementary Table 1 and 5), taxonomically classified into 72 putative species (ANI > 95\%) and 37 genera. No MAGs were classified as eukaryotes, and only $0.21 \%$ of the contigs in the MAGs with completeness $<70$ and contamination $>10 \%$ were assigned a likely eukaryotic origin. We thus tentatively conclude that eukaryotes are rare or undetectable in the Microcystis microbiome, as defined here. Only five AB species were shared among colonies from Canada and Brazil: Pseudanabaena sp. A06, Methylobacterium sp. A30, Roseomonas sp. A21, Burkholderia sp. A55 (a likely contaminant, as discussed below) and Gemmatimonas sp. A63 (Supplementary Fig. 2 ). Because certain low-abundance $A B$ might be present in a colony but fail to assemble, we mapped reads from each colony to a database of all MAGs and estimated $A B$ genome coverages. Using this approach, we found that each colony contained an average of six $\mathrm{AB}$ (genome coverage greater or equal to $1 \times$ ), with a range of 0 to 15 (Supplementary Fig. 3). We found no strict "core" of AB present in all colonies, either at the species or genus level. However, several genera were quite prevalent. These include Phenylobacterium (present in $73.40 \%$ of colonies), Roseomonas (70.64\%), Pseudanabaena (43.12\%), Rhodobacter (46.79\%), Methylobacterium (44.04\%), Rhodocyclaceae G1 (unclassified genus) (39.45\%), Rhodocyclaceae G2 (unclassified genus) (31.19\%), Chitinophagaceae (unclassified genus) (26.60\%), and Cytophagales (unclassified genus) (22.94\%). It is possible that a core Microcystis microbiome does exist, but includes rare $\mathrm{AB}$ that were not assembled into MAGs. To test this possibility, we used two alternative assembly-independent metagenomic read mapping approaches to define the microbiome: Kaiju and MIDAS (Methods). We could not find a core microbiome based on the reference MIDAS database, which contains more than 30,000 reference genomes classified into 5952 species. With Kaiju, which is based on NCBI Reference Sequences, we identified between 0 and 78 core species depending on the read count cutoff (Supplementary Fig. 4 and Supplementary Table 6). However, the core microbiome detected by Kaiju is likely due to misclassification of reads at the species level, which poorly matches the species classification based on MIDAS. For example, the inferred core species supported by at least 1000 reads by Kaiju (still less than $1 \times$ coverage of a typical bacterial genome) were Pseudomonas aeruginosa and an Acidobacteria species, which we consider likely contaminants. We therefore conclude that, at the resolution of reasonably deep metagenomic sequencing, a strict core microbiome does not exist.

To assess the evidence for phylosymbiosis, we first asked if different Microcystis genotypes had distinct colony microbiomes. The phylogeny illustrates how certain Microcystis genotypes appear to be preferentially associated with particular AB (Fig. 1). For example, Phenylobacterium and Methylobacterium were present in all sampled colonies of genotype G15, while Rhodobacter and Phenylobacterium occur in all colonies of genotype G01. These anecdotal patterns are borne out in statistical analyses of colony community structure, which show that Microcystis genotypes have significantly distinct microbiomes relative to the null model of random assignment of $\mathrm{AB}$ across genotypes (Fig. 3a). The genotype explains more variation in community structure (PERMANOVA on Bray-Curtis distances, $R^{2}=38.7 \%, P$ $<0.01$; Supplementary Table 7) than any other measured variable including $\mathrm{pH}\left(R^{2} \leq 5 \%\right)$ or temperature at the sampling site $\left(R^{2} \leq 5 \%\right)$, presence of microcystin $(m c y)$ genes in the genotype $\left(R^{2} \leq 5 \%\right)$, or location of sampling $\left(R^{2}=11.8 \%\right)$. Genotype was still the best explanatory variable when the analysis was performed on Lake Champlain samples only to exclude the effects of geography (Fig. 3b, PERMANOVA, $R^{2}=30.9 \%, P=0.001$ ).

A key piece of evidence for phylosymbiosis is not only for microbiomes to differ among host lineages, but for microbiome composition to change proportionally to host phylogeny. To test this, we converted the Microcystis host phylogeny into a distance matrix, which we correlated with the colony microbiome Bray-Curtis dissimilarity matrix. Consistent with phylosymbiosis, we found that variation in microbiome composition was correlated with the host phylogeny according to a Mantel test $(r=0.5, P=0.001)$, and further confirmed with Procrustean superimposition $(r=0.6, P=0.001)$ [36]. Similar evidence for phylosymbiosis was obtained using Kaiju or an expanded MIDAS reference genome database (Supplementary Table 8). While these alternative approaches yielded significant Mantel and Procrustes tests, the correlation coefficients were slightly lower than obtained with our set of 72 MAGs. This suggests that the alternative methods, which may be more sensitive to rare, unassembled taxa, likely add noise rather than strengthen the signal of phylosymbiosis. Taken together, these results provide strong support for phylosymbiosis in Microcystis colonies. 


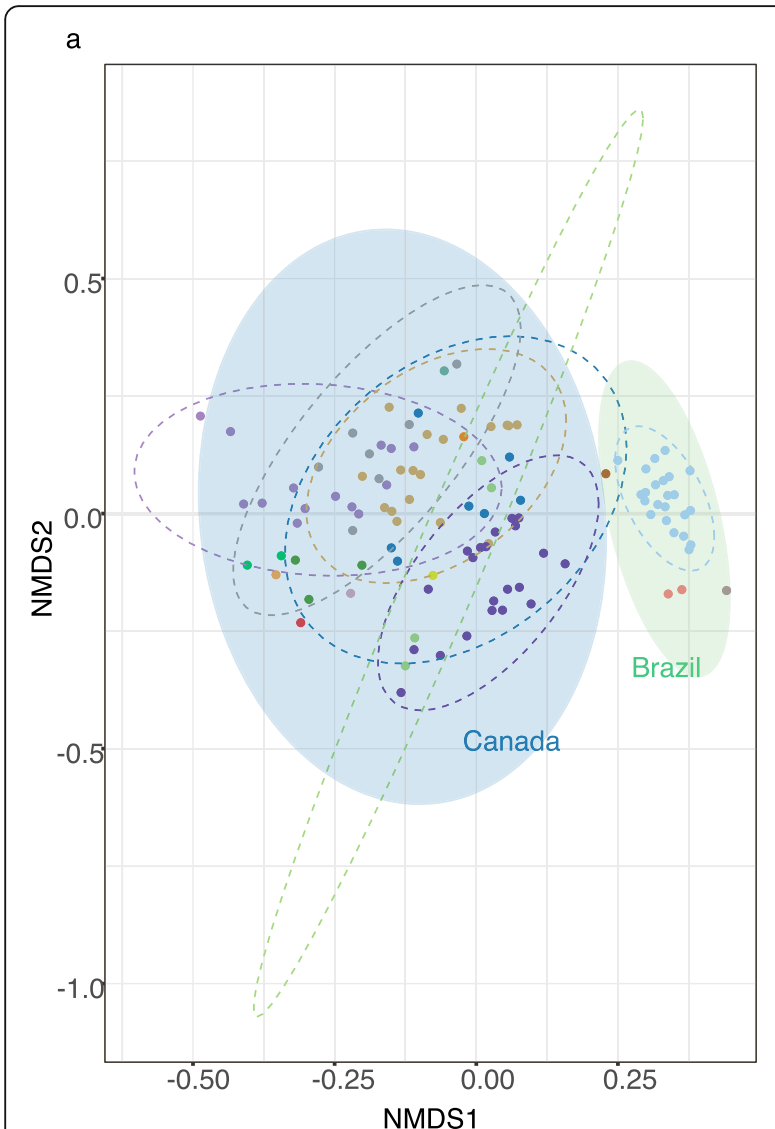

b

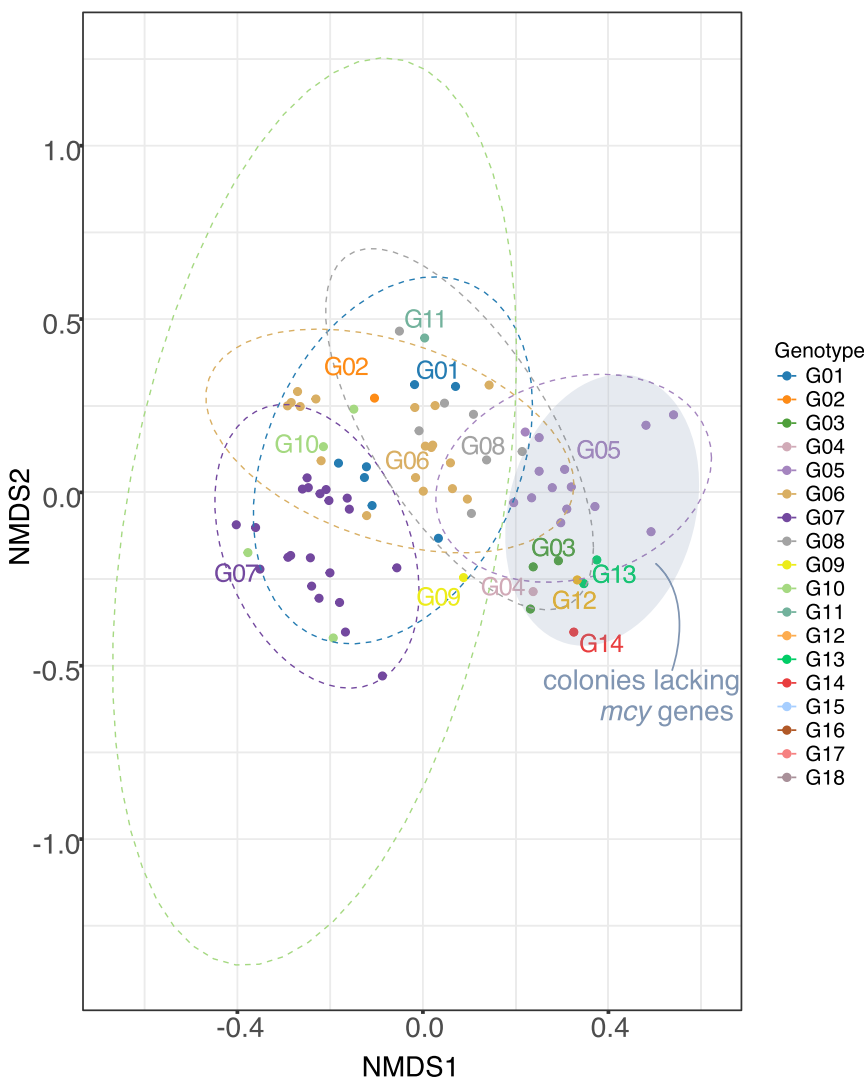

Fig. 3 Microcystis genotypes have distinct microbiomes. Nonmetric multidimensional scaling (NMDS) plots are based on the coverage of the nonMicrocystis metagenome-assembled genomes (MAGs) per colony (Bray-Curtis distance). a All samples, including those from Pampulha, Brazil and Lake Champlain, Canada. Ellipses show 95\% confidence intervals of $\mathbf{b}$ samples from Lake Champlain only (stress $=0.225$ ). The grey-shaded ellipse shows Microcystis colonies that do not encode the mcy cluster for microcystin toxin production

Microcystis genotype abundances vary over time in Lake Champlain and are correlated with prevalent members of the microbiome

Toxigenic and non-toxigenic genotypes of Microcystis are known to change in relative abundance within lakes over time [34, 37, 38]. More generally, to what extent different genotypes of Microcystis vary over time, along with their colony-associated bacteria, is less well studied. To investigate the Microcystis genotype diversity in metagenomes from Lake Champlain, we used a database of 15 single-copy taxonomic marker genes, which are mostly involved in translation, and ribosomal structure and biogenesis (Supplementary Table 9) [39]. This database was used to distinguish among the 14 Microcystis genotypes identified from colony sequencing (Methods), and to estimate their relative abundance in 72 metagenomes sampled from filtered lake water during the summer months of 2006 to 2018 (Supplementary Fig. 5). It is possible that these 14 genotypes do not represent the total genotypic diversity of Microcystis occurring in the lake. However, mapping metagenomic reads from the lake to these genotypes with a 99\% sequence identity threshold allowed us to recover $93.5 \%$ of Microcystis reads. Microcystis reads were defined at $>96 \%$ sequence identity to the Microcystis reference genome M083S1 (Methods). Therefore, the 14 genotypes are representative of the vast majority of Microcystis diversity captured in lake metagenomes.

Using a distance-based redundancy analysis (dbRDA), we estimated the effects of total phosphorous, total nitrogen, dissolved phosphorous, dissolved nitrogen, mean temperature and time (years, months and season) on the Microcystis genotype community composition in the 42 Lake Champlain metagenomes with complete metadata, and with Microcystis genome coverage greater or equal to $1 \times$. Microcystis genotype diversity was best explained by yearly temporal variation $\left(\mathrm{dbRDA}, R^{2}=51.1 \%, P<0.01\right.$; PERMANOVA, $R^{2}=$ 47.9\%, $P<0.01$; Supplementary Fig. 6). Years did not differ significantly in their dispersion (PERMDISP $P>$ 0.05; Supplementary Table 7). Environmental variables such as nitrogen and phosphorus did not have a 
significant effect on the community composition. In a shorter time series (April to November of 1 year) in Pampulha, a more diverse community of four Microcystis genotypes eventually became dominated by one genotype (G15) encoding the mcy toxin biosynthesis gene cluster (Supplementary Fig. 7). However, more extensive sampling is required to estimate the effect of other environmental variables (e.g., phosphorus) on the community composition in Brazil.

Similarly to Microcystis genotypes, the composition of $\mathrm{AB}$ in Lake Champlain also varied significantly across years (dbRDA, $R^{2}=44.3 \%, P<0.01$; PERMANOVA, $R^{2}$ $=43.5 \%, P<0.01$; Supplementary Fig. 8 ). We asked if the presence of dominant Microcystis genotypes could explain the variation in the $\mathrm{AB}$ community composition. A significant effect of the genotype was observed using PERMANOVA $\left(R^{2}=31.5 \%, P<0.01\right)$, but not using dbRDA $\left(R^{2}=0.012, P>0.05\right)$. Years and Microcystis genotypes were the best explanatory variables for $\mathrm{AB}$ composition; however, their dispersions were significantly different $(P<0.01)$ making the PERMANOVA results difficult to interpret. In addition, the $\mathrm{AB}$ community sampled from metagenomes includes both free-living and colony-attached $\mathrm{AB}$, possibly adding noise to any signal of Microcystis genotypes selecting for specific $\mathrm{AB}$ within colonies. While these results are statistically somewhat ambiguous, they are generally consistent with phylosymbiosis between Microcystis and its microbiome.

We further hypothesized that the most prevalent $A B$ in the Microcystis microbiome should co-occur with Microcystis in lake metagenomes. In contrast, they should not co-occur with another cyanobacterium frequently observed in Lake Champlain, Dolichospermum, which serves as a negative control. We first estimated normalized read counts and coverage of Microcystis and Dolichospermum in the 72 metagenomes from the Lake Champlain time series (Supplementary Fig. 9). We then estimated the Spearman correlations between Microcystis or Dolichospermum and each $\mathrm{AB}$ species or genus. The two cyanobacteria were weakly correlated with each other across the environmental metagenomes (Spearman's $\rho=0.29$ and $Q$ value $=0.027$ ). As expected, the nine most prevalent $\mathrm{AB}$ genera in the Microcystis microbiome were strongly correlated with Microcystis (Spearman's $\rho>0.7, Q$ value $<0.001$ ), and only weakly with Dolichospermum (Spearman's $\rho<0.4, Q$ value $>0.001$ ) with the exception of Phenylobacterium (Spearman's $\rho=$ $0.47, Q$ value $<0.001$ ) which is nevertheless more strongly associated with Microcystis (Supplementary Figs. 10 and 11). The positive correlation between the most prevalent $\mathrm{AB}$ genera and Microcystis was also supported using an alternative correlation method, SparCC, which corrects for compositional effects in the relative abundance data (SparCC $r>0.4, Q$ value < 0.05) (Supplementary Table 10 and Supplementary Fig. 12). These significant positive correlations are consistent with close interaction between Microcystis and the most prevalent genera in their microbiome. Genera found at lower prevalence in Microcystis colonies (e.g., Phycisphaerales bacterium (unclassified genus) and Telmatospirillum) were poorly correlated with both Microcystis and Dolichospermum, suggesting weaker or transient associations (Supplementary Table 10 and Supplementary Fig. 10a).

Another $\mathrm{AB}$ belonging to the genus Burkholderia was quite prevalent in colonies but poorly correlated with Microcystis in metagenomes (present in the $40.37 \%$ of the colonies; Spearman's $\rho=-0.16, Q$ value $=0.343$ ) suggesting contamination of colonies rather than a true ecological association. The genomes within this Burkholderia species showed nucleotide identity greater than 99\% and short phylogenetic distances (0.0001), and was found in both Canadian and Brazilian colonies, suggesting a clonal lab contaminant rather than a batch or sampling effect. However, this was the only signal of contamination, suggesting that most of the other data reflect true associations.

Having already provided general support for phylosymbiosis, we sought to illustrate examples of associations between specific Microcystis genotypes and specific AB species. For example, Rhodocyclaceae bacterium G2 A13 was better correlated with genotype G05 than other Microcystis genotypes (Supplementary Fig. 13), consistent with the prevalence of this $A B$ in 13 out of 14 colonies of genotype G05. In contrast, genotype G10 was poorly correlated with certain species within the genera Roseomonas and Methylobacterium (Spearman's $\rho<$ $0.38, Q$ value $>0.001)$. This illustrates how certain Microcystis genotypes have strong preferences for certain $\mathrm{AB}$, while being unselective for others.

\section{Signatures of co-speciation between Microcystis and members of its microbiome}

Phylosymbiosis can arise due to vertical inheritance of microbiomes, or horizontal acquisition of microbiomes at each generation, provided that host lineages preferentially "match" with distinct microbiomes [24]. To assess the evidence for vertical inheritance of Microcystis AB, we used ParaFit to test for similarity between the Microcystis phylogeny and the phylogenies of the nine most prevalent $A B$ genera strongly correlated with Microcystis but not with Dolichospermum in Lake Champlain (Supplementary Fig. 11). Each of these genera was represented by at least 12 high-quality draft genomes and was found in at least five different Microcystis genotypes. Significant cophylogenetic signal suggests co-speciation of hosts and symbionts, consistent with a relatively long evolutionary history of association (e.g., vertical descent). 
The ideal signal of co-phylogeny would be exactly congruent tree topologies for Microcystis and AB. In reality, we found tree topologies that were significantly similar (according to the ParaFit test), despite being non-identical. For example, Roseomonas, the second most prevalent $\mathrm{AB}$ genus in colonies, and Rhodobacter, the third most prevalent, had significant signatures of co-phylogeny (Fig. 4), while Phenylobacterium and Chitinophagaceae were borderline cases (Table 1). Overall, there was no clear tendency for stronger co-phylogeny with more prevalent $A B$, or with $\mathrm{AB}$ most correlated with Microcystis over time in Lake Champlain metagenomes (Table 1). However, such tendencies would be hard to discern in this relatively small sample size. As expected, the suspected contaminant Burkholderia A55 (Burkholderia cepacia) present in $40.37 \%$ of colonies, was poorly correlated with the presence of Microcystis in environmental metagenomes $(r=-0.16, Q$ value $=0.343)$, with no signal of co-phylogeny $(P$ value $=0.732)$. Although co-phylogenetic signal was detectable in at least two of the most prevalent $A B$, the phylogenies are not identical (Fig. 4), suggesting a mixture of vertical and horizontal transmission. Even if horizontal transmission of $\mathrm{AB}$ among Microcystis lineages is likely, some degree of non-random host-microbiome matching must be occurring to explain the co-phylogenetic signal.

\section{Horizontal gene transfer (HGT) between Microcystis and its associated bacteria}

Unrelated bacteria sharing a common environment, such as the human gut, are known to engage in frequent horizontal gene transfer [40]. We hypothesized that Microcystis would also exchange genes with members of its microbiome, which share a similar physical niche-the colony milieu - for at least some period of time. We began by using a simple heuristic to look for similar gene sequences ( $\geq 99 \%$ amino acid identity) occurring in the Microcystis genome and at least one $\mathrm{AB}$ genome, as a

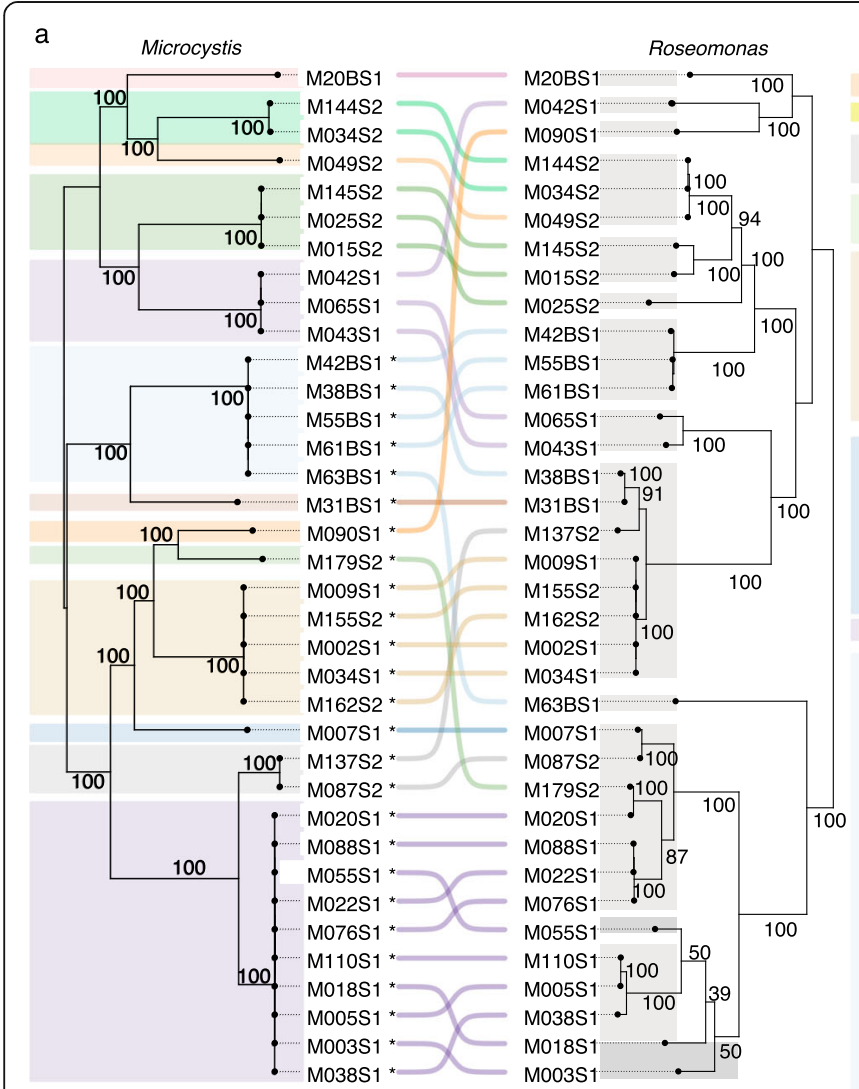

Tree scale: 0.01

Tree scale: $0.01 \mapsto$

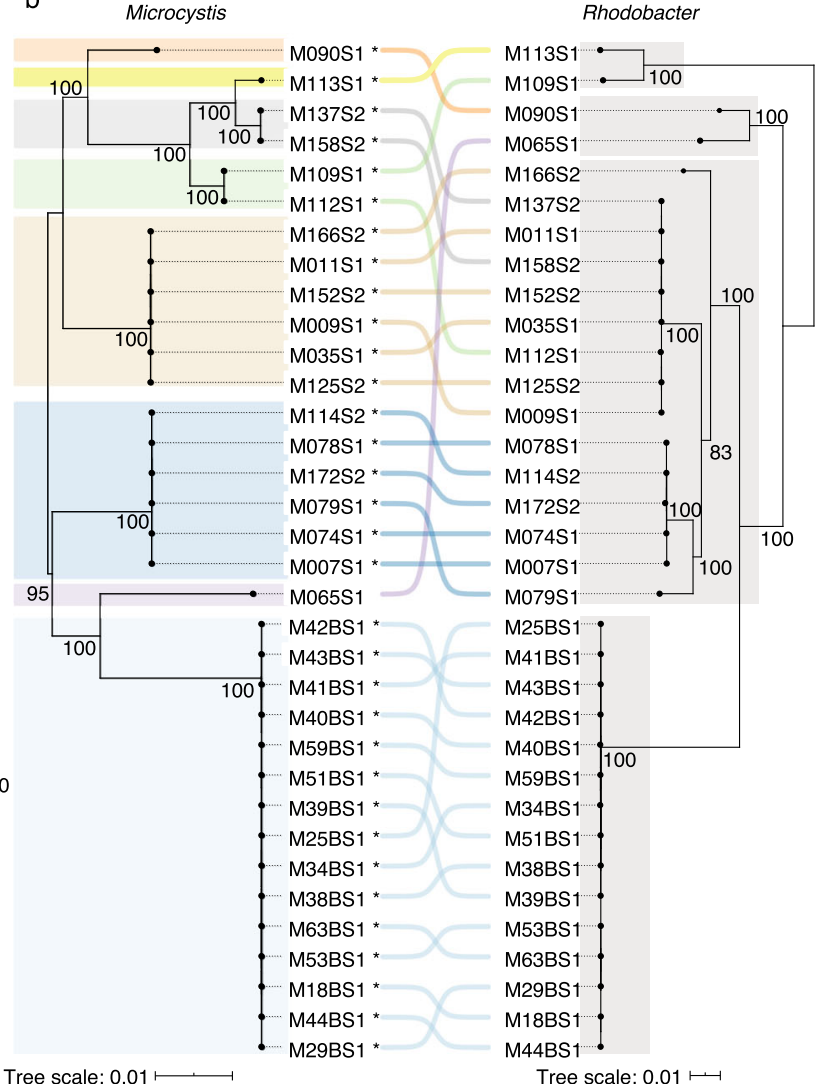

Fig. 4 Co-phylogeny between Microcystis and two prevalent associated bacteria. a Roseomonas and $\mathbf{b}$ Rhodobacter core genome phylogenies were compared to the Microcystis core phylogeny. The lines between the two phylogenies connect genomes coming from the same Microcystis colony. The phylogenetic trees for Microcystis, Roseomonas and Rhodobacter were based on 706, 135 and 470 core genes, respectively. The different Microcystis genotypes are highlighted in colour, and the Roseomonas or Rhodobacter species in gray. The asterisks indicate the presence of the mcy cluster. While in a perfect co-phylogeny, the tree topologies of host and symbiont are identical; in these two comparisons, the cophylogenetic similarity is imperfect but greater than expected by chance (ParaFit Global test, $P$ value $<0.01$ ) 
Table 1 Co-phylogeny analysis between Microcystis and the nine most prevalent associated bacterial genera within the Microcystis microbiome

\begin{tabular}{|c|c|c|c|c|c|}
\hline $\begin{array}{l}\text { Associated } \\
\text { bacteria (AB) } \\
\text { genus }\end{array}$ & $\begin{array}{l}\text { Number of } \\
\text { species per } \\
\text { genus }\end{array}$ & $\begin{array}{l}\text { Number of } A B \text { genomes } \\
\text { used in the phylogeny }\end{array}$ & $\begin{array}{l}\text { Prevalence of } A B \text { in colonies } \\
\text { from Canada and Brazil }\end{array}$ & $\begin{array}{l}\text { Correlation with Microcystis in } \\
\text { Canada metagenomes }\left(r^{2}\right)\end{array}$ & $\begin{array}{l}\text { ParaFit } \\
\text { test } \\
(P \\
\text { values })\end{array}$ \\
\hline Phenylobacterium & 5 & 60 & $73.40 \%$ & $0.759 *$ & $\begin{array}{l}0.072 \\
(0.008)\end{array}$ \\
\hline Roseomonas & 13 & 36 & $70.64 \%$ & $0.835 *$ & $\begin{array}{l}0.009^{* *} \\
(0.001)\end{array}$ \\
\hline Rhodobacter & 4 & 34 & $46.79 \%$ & $0.779 *$ & $\begin{array}{l}0.0018^{* *} \\
(0.0002)\end{array}$ \\
\hline Methylobacterium & 3 & 29 & $44.04 \%$ & $0.809 *$ & $\begin{array}{l}0.729 \\
(0.081)\end{array}$ \\
\hline Pseudanabaena & 2 & 20 & $43.12 \%$ & $0.766 *$ & $\begin{array}{l}0.153 \\
(0.017)\end{array}$ \\
\hline $\begin{array}{l}\text { Rhodocyclaceae } \\
\text { bacterium G1 }\end{array}$ & 2 & 19 & $39.45 \%$ & $0.769 *$ & $\begin{array}{l}0.225 \\
(0.025)\end{array}$ \\
\hline $\begin{array}{l}\text { Rhodocyclaceae } \\
\text { bacterium } \mathrm{G} 2\end{array}$ & 2 & 21 & $31.19 \%$ & $0.776 *$ & $\begin{array}{l}5.355 \\
(0.595)\end{array}$ \\
\hline $\begin{array}{l}\text { Chitinophagaceae } \\
\text { bacterium }\end{array}$ & 3 & 22 & $26.60 \%$ & $0.795 *$ & $\begin{array}{l}0.081 \\
(0.009)\end{array}$ \\
\hline $\begin{array}{l}\text { Cytophagales } \\
\text { bacterium }\end{array}$ & 3 & 16 & $22.94 \%$ & $0.740 *$ & $\begin{array}{l}0.702 \\
(0.078)\end{array}$ \\
\hline
\end{tabular}

* Significant correlation coefficients $(Q<0.01)$

** Significant $P$ values $(P<0.01)$ (Bonferroni correction). Uncorrected $P$ values are shown between parentheses

proxy for relatively recent HGT events. Genome assembly and binning could affect this analysis by misplacing identical sequences either in Microcystis or in an AB genome, but not in both. To reduce this possible bias, we only considered a gene to be involved in HGT if it was present in at least four genomes (which would be unlikely to occur due to binning errors alone). We identified a total of 1909 genes involved in HGT between Microcystis and one of seven AB species: Pseudanabaena A06, Pseudanabaena A07, Burkholderiales bacterium G3 A12, Rhodocyclaceae bacterium G2 A13, Chitinophagaceae bacterium A08, Cytophagales bacterium A04 and Cytophagales bacterium A05. Compared to the Microcystis core genes, these candidate HGTs are enriched in functions related to secondary metabolite biosynthesis, replication and recombination, and defense mechanisms (Fig. 5). As a control, we repeated the HGT inference using the likely contaminant Burkholderia A55 genome instead of Microcystis. We identified 558 putative HGT events, of which 523 involved species were not found to engage in HGT with Microcystis: Methylobacterium A30, Rhodocyclaceae bacterium G1 A54 and Cupriavidus A44. This suggests that Microcystis engages in more HGT with its microbiome than a random expectation (i.e., with a contaminant genome), and allows us to conservatively estimate the false-positive rate of HGT detection at $523 /(523+1909)$, or $22 \%$. Despite the significant noise, we expect the broad gene functional categories and specific AB involved in HGT with Microcystis to be relatively robust.
As an additional validation of our HGT heuristic, we used Metachip, which uses phylogenetic incongruence in addition to a sequence identity threshold [41]. Metachip identified the same seven $\mathrm{AB}$ genera involved in HGT with Microcystis based on our simple heuristic, except for Rhodocyclaceae bacterium G2. Metachip is much more conservative, identifying only 46 gene families involved in HGT (Supplementary Table 11). Of these gene families, 31 were also identified by our heuristic method, suggesting they are high-quality candidates.

\section{Cellular functions encoded by members of the Microcystis microbiome}

In contrast to genes shared by HGT, there may be a genetic division of labour between Microcystis and its microbiome, which would then be expected to encode different and complementary sets of gene functions. To compare these gene functions, we first characterized orthologous genes using the Kyoto Encyclopedia of Genes and Genomes (KEGG) orthologues (KO) in both Microcystis and its microbiome. We then used ANTISMASH [42] to identify gene clusters involved in the biosynthesis of cyanopeptides and other pathways of interest. We further validated the presence of biosynthetic pathways like biotin, cobalamin, nitrogen fixation and carotenoids with gapseq [43]. As expected for distantly related bacteria, Microcystis genotypes and $\mathrm{AB}$ encode distinct sets of gene functions based on the presence/absence of annotated genes (Supplementary 


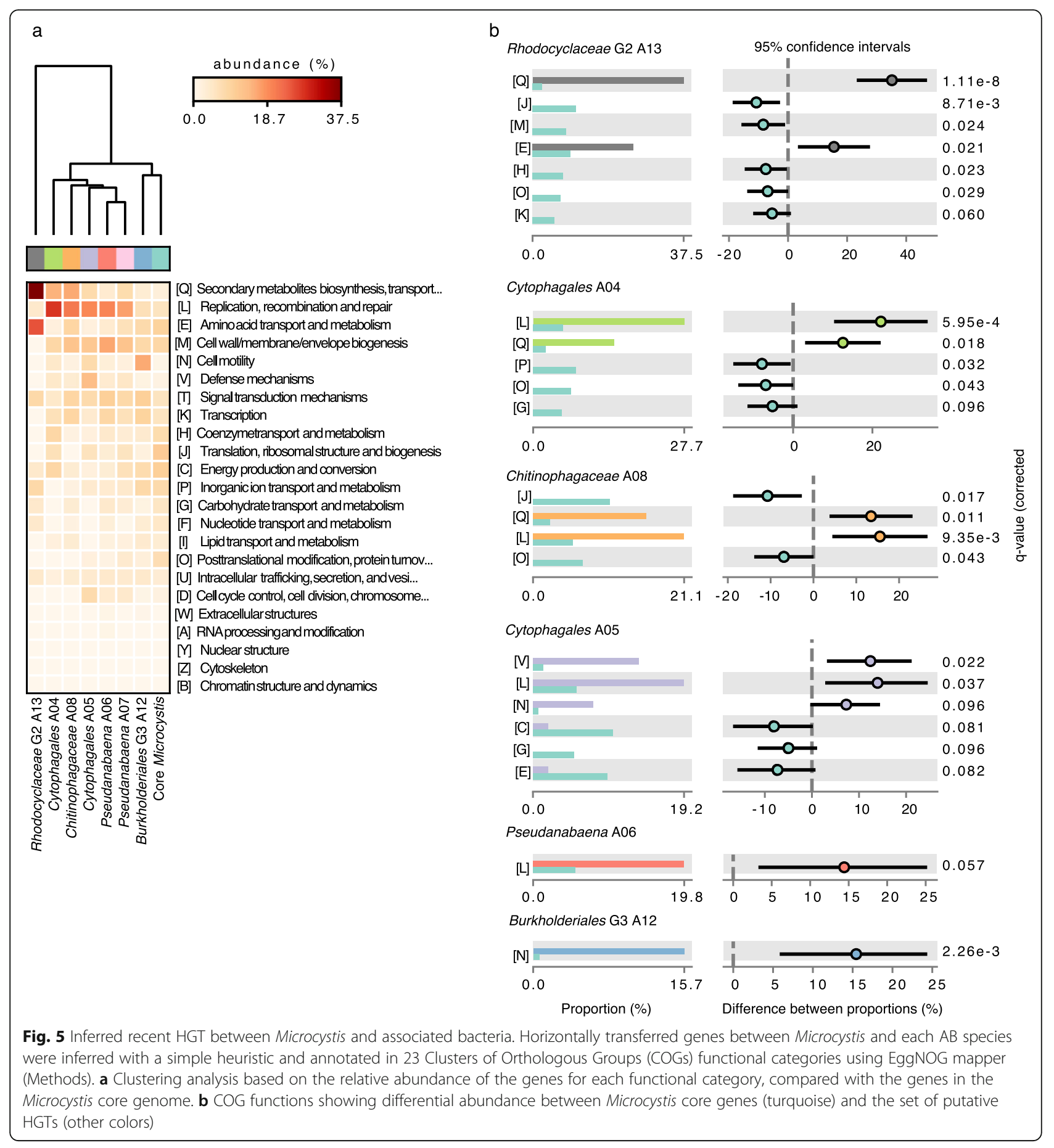

Fig. 14). Bacteria from the same Phylum tend to cluster together in terms of their functional gene content. For example, Microcystis clusters with its fellow cyanobacteria Pseudanabaena, while Bacteroidetes (i.e., Cytophagales bacterium and Chitinophagaceae bacterium) formed a distinct cluster (Supplementary Fig. 14).

Roseomonas and Rhodobacter, which show cophylogeny with Microcystis but appear not to engage in significant amounts of HGT, are prime candidates for functional complementarity to have evolved and be maintained with high partner fidelity. Both these genera contain carotenoid biosynthesis pathways different from those found in Microcystis genomes (Supplementary Table 12). For example, neurosporene, spirilloxanthin and spheroidene pathways are present in Rhodobacter but not in Microcystis. Carotenoid pigments like zeaxanthin are 
generally produced by Microcystis for their photoprotective properties and their capacity to improve the efficiency of photosynthesis [44]. Indeed, in our Microcystis genomes, we found the complete pathways for the biosynthesis of zeaxanthin, echinenone and intermediaries in the biosynthesis of carotenoids like trans-lycopene. It is tempting to speculate that the Microcystis microbiome could be involved in the production of additional carotenoids that could provide additional photoprotection under certain environmental conditions; this deserves further study. Certain AB (i.e., Roseomonas, Rhodobacter and Methylobacterium) also encoded genes involved in anoxygenic photosynthesis (absent in Microcystis) and the transport of rhamnose, D-xylose, fructose, glycerol and aglucoside (Supplementary Table 13), which could also complement the metabolic repertoire of Microcystis [16].

To test for potential metabolic interactions between Microcystis and its microbiome, we reconstructed genome-scale metabolic models with CarveMe [45] and used SMETANA [46] to identify exchanged metabolites that are essential for the survival of the community (Methods). We performed these analyses on Microcystis genotype $\mathrm{G} 01$ and its microbiome of five $\mathrm{AB}$, which are among the most prevalent across the genus. This analysis suggests that Microcystis is a major recipient (but not a donor) of metabolites from $\mathrm{AB}$, including amino acids and nutrients (Supplementary table 14A). As a negative control, we analyzed the interactions between Microcystis and taxa that did not co-occur (based on SparCC and Spearman correlation results), yielding no metabolic exchanges inferred by SMETANA. Microcystis is unable to fix nitrogen, but it contains pathways to assimilate nitrate and ammonia. Previous studies have suggested that Microcystis might rely on its microbiome for nitrogen [16, 47]. Although we found no complete pathways for nitrogen fixation in $\mathrm{AB}$ genomes, metabolic reconstruction suggests that Methylobacterium, Roseomonas and Rhodobacter might provide Microcystis with nitrate, nitrite and ammonium (Supplementary Table 14A). In a second SMETANA "global" analysis of species pairs, we found that Roseomonas is the $\mathrm{AB}$ with the lowest metabolic resource overlap (MRO score) and the highest metabolic interaction potential (MIP score), suggesting metabolic complementarity (Supplementary Table 14B). Roseomonas was the only AB with a lower MRO score and a higher MIP score than observed in negative controls (Supplementary Table 14C). Together, these results suggest the potential for significant metabolic exchange between Microcystis and its microbiome, particularly Roseomonas.

\section{Discussion}

By combining single colony sequencing and metagenome analysis, we explored the genetic diversity of both
Microcystis and its microbiome, and their variation over time in Lake Champlain, Canada and the Pampulha reservoir in Brazil. We revealed a higher diversity of Microcystis genotypes than previously described [48], and a strong signal of phylosymbiosis between the host and its microbiome. Despite the absence of a strict core microbiome, the overall microbiome community composition tends to change proportionally along the Microcystis host phylogeny-a hallmark of phylosymbiosis [24]. Although some members of the microbiome might be loosely associated, some-notably Roseomonas and Rhodobacter-appear to be relatively stable over evolutionary time (taking phylogenetic branch length as a rough proxy for time). These two genera have been previously reported to be correlated with Microcystis in environmental samples $[49,50]$. Whether these associations are beneficial to one or both partners remain to be seen.

There has been some debate about whether Microcystis colonies form by clonal cell division, or by aggregation of (potentially distantly related) cyanobacterial cells $[21,51]$. Our results suggest that clonal cell division is more likely, based on our observation of much greater genetic variation in the Microcystis genome between than within colonies of the same genotype. This finding applies to the relatively large colonies we sampled from eutrophic lakes, and results could differ for smaller colonies, under oligotrophic conditions [32], or under strong grazing predation, which could affect colony structures [52]. Because DNA extraction is more likely to have failed for smaller colonies, our collection likely underrepresented smaller colonies, which could represent distinct genotypes. However, 93.5\% of Microcystis metagenomic reads from Lake Champlain were recruited to our collection of colony genomes at $99 \%$ nucleotide sequence identity, suggesting that the majority of natural Microcystis diversity is represented in our sample of colonies. Of course, these results are specific to Lake Champlain and Pampulha, and should be replicated in other lakes under different environmental conditions (e.g., oligotrophic lakes).

Phylosymbiosis and co-speciation appear to be relatively common and strong in mammalian gut microbiomes [24, 25], and even in the more environmentally exposed coral microbiome [24, 25]. It is unclear if such tight and evolutionarily stable associations would apply to Microcystis and its $\mathrm{AB}$, or if more transient interactions would prevail. While the idea of a Microcystis microbiome has been suggested previously based on bulk metagenomic and amplicon sequencing from lakes $[16,53]$, here we refine the Microcystis microbiome concept beyond co-occurrence patterns to physical association within a colony. We found that the most prevalent $\mathrm{AB}$ from individual Microcystis colonies also tend to co- 
occur with Microcystis over time in Lake Champlain. The composition of the microbiome varies along the Microcystis phylogenetic tree, consistent with phylosymbiosis and relatively long-term evolutionary associations. At least two $A B$ show a significant co-phylogenetic signal, suggesting co-speciation with Microcystis. Therefore, although possibly not as strong as in mammals or even coral, cophylogeny is a feature of at least certain members of the Microcystis microbiome, yet co-phylogeny is unlikely to explain most of the signal of phylosymbiosis. Alternatively, phylosymbiosis can arise as a consequence of shared biogeography between hosts and microbiomes [54], and we do observe distinct microbiomes in Brazil and Canada. However, we found evidence for phylosymbiosis within a single lake in Canada, suggesting that factors other than biogeography-such as host-microbiome trait matchingare likely at play.

As expected for distantly related bacteria, Microcystis and its $\mathrm{AB}$ encode different functional gene repertoires, some of which could be complementary and mutually beneficial. We found that both Microcystis and $\mathrm{AB}$ have biosynthetic functions for a wide range of carotenoids. Metabolic pathways for carotenoids in Microcystis differed from those found in AB. Carotenoids act as antioxidants and may broaden the photosynthetic light absorption spectrum $[55,56]$. In a previous study, heterotrophic bacteria producing carotenoid pigments showed higher survival to environmental stressors (e.g., solar radiation) than their unpigmented counterparts [57]. Further studies are required to understand whether the additional carotenoid pathways in the $A B$ enhance the host photo protection.

Horizontal gene transfer (HGT) is relatively common in bacteria, and may occur among unrelated bacteria [58] particularly when they share an ecological niche such as the human gut [40]. Microcystis is physically associated with its microbiome for at least part of the colony life cycle, and we hypothesized that HGT could occur within colonies. Using two methods to detect HGT, we found evidence for gene transfers between Microcystis and at least six different species of $\mathrm{AB}$ : two species of Pseudanabaena, two Cytophagales, one Burkholderiales, and one Chitinophagaceae species. Certain gene functions, including secondary metabolite biosynthesis, replication and recombination, and defense mechanisms, are particularly prone to HGT between Microcystis and its microbiome, and the ecological functions of these genes deserves further study. Notably, prevalent $\mathrm{AB}$ with evidence of co-phylogeny with Microcystis (Roseomonas and Rhodobacter) shared relatively few (less than seven) HGT events with Microcystis. This counter-intuitive result could be explained if these cophylogenetic associations are relatively ancient, but our HGT detection is biased toward recent events.
Alternatively, it is possible that HGT is more likely among less intimately associated $\mathrm{AB}$, whereas more intimate associations would select for only one, but not both partners, to encode the gene. This would also require that metabolites are shared between partners. To explain this result, we hypothesize that such long-term associations might favour the loss of redundant genes, as predicted by the Black Queen Hypothesis [59]. In other words, a gene needs to be encoded by only one partner, provided that gene products or metabolites are shared between partners. Such metabolic interdependencies appear to be relatively strong between Microcystis and Roseomonas. Therefore, even if HGT does occur between partners, we would not expect to find the same gene redundantly encoded in both partners. These evolved co-dependencies would further reinforce partner fidelity and could help explain the co-phylogenetic signal between them.

\section{Conclusions}

Overall, our results provide evidence for ecoevolutionary associations between Microcystis and its microbiome. Some members of the microbiome may be more tightly associated than others, and based on their gene content, we hypothesize that they might provide complementary functions to Microcystis. Such complementary functions can be encoded by a variety of species, such that there is no strict species- or genus-level core microbiome. These hypotheses could be tested in experimental co-cultures, which have recently shown how the Microcystis microbiome can alter its competitive fitness against eukaryotic algae [60]. Such experiments could be extended to the combinations of Microcystis genotypes and $\mathrm{AB}$ which we have shown to be intimately associated in nature.

\section{Materials and methods \\ Sample collection and DNA extraction for colonies and metagenomes}

To access the genomic diversity of Microcystis in Lake Champlain and Pampulha reservoir, 346 individual Microcystis colonies were isolated across the bloom season in 2017 and 2018 (July to October in Quebec, Canada ( $45^{\circ} 02^{\prime} 44.86^{\prime \prime} \mathrm{N}, 73^{\circ} 07^{\prime} 57.60^{\prime \prime} \mathrm{W}$ ) and April to November in Minas Gerais, Brazil (19 $55^{\circ} 09^{\prime \prime} \mathrm{S}$ and $43^{\circ}$ $\left.56^{\prime} 47^{\prime \prime} \mathrm{W}\right)$ ) during 2018. Colonies were isolated from surface water samples $(\sim 50 \mathrm{~cm}$ depth) after concentration using a plankton net (mesh size $20 \mu \mathrm{m})$. One litre of concentrated water was collected and stored at $4{ }^{\circ} \mathrm{C}$ for a maximum of $36 \mathrm{~h}$ until colony isolation. Colonies were isolated using micropipes, sterile medium ( $\mathrm{Z} 8$ medium) and a microscope (Nikon E200 Eclipse). Each colony was washed 15-20 times using sterile Z8 medium and stored at $-80{ }^{\circ} \mathrm{C}$ until DNA extraction. The DNA 
extraction was performed directly on each colony using the ChargeSwitch ${ }^{\circ}$ gDNA Mini Bacteria Kit. Two additional steps were added to ensure the rupture of the Microcystis colonies and cells (see Supplementary Methods). Briefly, each colony was added to a tube containing $50 \mathrm{mg}$ of beads (PowerBead tubes, glass $0.1 \mathrm{~mm}$, Mo-bio), incubated with lysis solutions, and then vortexed using the TissueLyser LT (Qiagen) for $3 \mathrm{~min}$ at 45 oscillations per second. The tube was then centrifuged for $1 \mathrm{~min}$ at $9000 \mathrm{rcf}$. This procedure yielded DNA for 109 colonies (Supplementary Figs. 15 and 16), sequenced as described below. Matched water samples were collected at the same place and time as colonies, spanning 16 time points (Supplementary Table 15). Water temperature and $\mathrm{pH}$ were also measured at each sampling point.

For metagenomic sequencing, a total of 72 lake water samples were collected over 10 years (2006 to 2018) during the ice-free season (April to November) from the photic zone of Missisquoi Bay at two different sites (littoral and pelagic) of Lake Champlain, Quebec, Canada ( $\left.45^{\circ} 02^{\prime} 45^{\prime \prime} \mathrm{N}, 73^{\circ} 07^{\prime} 58^{\prime \prime} \mathrm{W}\right)$. Lake water was filtered, and DNA was extracted using a Zymo Kit (Zymo, D4023) as described previously [61]. The filtration was performed the same day of the sampling, using between 50 and $250 \mathrm{~mL}$ of water samples, depending on the amount of biomass, onto $0.2 \mu \mathrm{m}$ hydrophilic polyethersulfone membranes (Millipore, Etobicoke, Ontario, Canada). Samples were obtained at relatively low frequency between 2006 and 2016, and at higher frequency (approximately weekly or more often) during bloom periods between 2015 and 2016 (Supplementary Table 3). Note that no colonies were isolated from 2006 to 2016. Water samples corresponding to six sampling points from Minas Gerais Brazil were also collected for DNA extraction and metagenome sequencing. Environmental variables were measured for each sample. Sample water were collected $(50 \mathrm{~mL})$ for measuring nutrients $(\mathrm{DN}$, DP, TP and TN), except for the samples from Brazil (Supplementary Table 3) [61].

\section{DNA sequencing of single colonies and metagenomes}

DNA extracted from Microcystis single colonies was sequenced using the Illumina HiSeq 4000 platform with 150 bp paired-end reads. The sequencing libraries (with average fragment size $360 \mathrm{bp}$ ) were prepared using the NEB (New England Biolabs ${ }^{\circ}$ ) low input protocol. The DNA extracted from filtered bulk lake water for each sampling point (2017 and 2018) from Canada and Brazil were sequenced using Illumina NovaSeq 6000 S4 platform with $150 \mathrm{bp}$ paired-end reads. The earlier lake water samples from a previous long-term experiment in Lake Champlain (2006 to 2016) were sequenced using Illumina Hiseq2500 with 125 paired-end reads (Supplementary Table 3). The quality score and number of reads per sequenced sample are reported in Supplementary Table 16. Quality scores were calculated using the program fastp (v0.20.1) [62].

\section{Metagenome assembly and genome binning}

For each individual Microcystis colony, the sequencing reads were filtered and trimmed using Trimmomatic (v0.36) [63] then assembled with MEGA-HIT (v1.1.1) [64], producing contigs belonging to both Microcystis and $A B$ (see Supplementary Fig. 17 showing the distribution size of the contigs). We performed manual binning on each individual colony assembly separately using Anvi'o (v3.5) with default parameters, only including contigs larger than $2500 \mathrm{bp}$, as described previously [31, 65]. This contig size cutoff of $2500 \mathrm{bp}$ improves binning based on k-mer frequencies, and is standard for metagenomic analyses of microbial communities from diverse environments [66-68]. Briefly, for each sample, we generated a contig database, and the reads were mapped back to contigs using bowtie 2 (v2.3.4.3) [69]. The contigs were interactively manually curated in Anvi'o based on the read coverage from the mapping, tetranucleotide frequency, GC content and taxonomic annotations obtained from centrifuge (v1.0.3) [70], and using the program 'anvi-profile' as implemented in Anvi'o [65]. The quality of each resulting MAG was estimated using Anvi'o and based on a collection of 139 bacterial single-copy core genes [33, 65]. From the 109 colonies, 500 MAGs were identified (defined as having completeness $\geq 70 \%$ and contamination $\leq 10 \%$ as in Lee et al. [71]) (Supplementary Tables 1 and 5). We used the program dRep (v3.2.0) with default parameters to investigate the presence of redundant MAGs in each colony metagenomes [72]. However, within an individual colony, we found no redundant MAGs. MAGs were annotated using Prokka (v1.14.0) [73]. Pairwise average nucleotide identity (ANI) values between genomes were estimated using pyani (v0.2.8) [74, 75]. MAGs were classified into species-level taxonomic groups at a threshold of ANI $\geq 96 \%$ (Supplementary Tables 5 and 17). The MAGs were assigned species and genus names using BLASTp of the recA and RpoB proteins against the NCBI database, and refined using the Genome Taxonomy Database Toolkit (GTDB-Tk) (v1.0.2), which uses a set 120 universal bacterial gene markers [76]. To assess the presence of eukaryotic microbes, the contigs in the uncomplete or MAGs with completeness $<70 \%$ and contamination $>10 \%$ were classified taxonomically using the program CAT (v5.2.3) (Supplementary Table 18) [77].

For each taxonomic group, we selected at least two representative sequence types (for a total of 138 genomes), from which we inferred a maximum 
likelihood phylogenetic tree based on the core gene alignment using RAxML (v8.2.11) [78]. The core genome was estimated using panX (v1.5.1). Core genes were defined as those genes present in at least the $80 \%$ of sampled genomes (e value < 0.005) [79]. Each of the resulting 62 core genes was alignment using muscle (v3.8.3) [80]. Filter.seqs from mothur (v1.41.3) was used to remove the gaps per each gene alignment [81]. Individual alignments were concatenated into a single alignment (16,400-bp long) input into RAxML. For the phylogenetic reconstruction, we used a GTR-GAMMA substitution model with 100 bootstraps, and best maximum likelihood tree inference.

\section{Assessment of the Microcystis genotype diversity in freshwater colonies}

A core genome was also estimated for the 109 Microcystis genomes and 122 NCBI references genomes (Supplementary Table 1 and 19). The resulting alignment of the 115 core genes was degapped (68,145-bp long) and used to infer an ML phylogeny using RAxML as we described previously. Two outgroups (Anabaena variabilis ATCC29413 and Synechocystis sp. PCC6803) were included. Based on ANI values greater or equal to $99 \%$, the monophyletic clades of Microcystis genomes were classified into 18 genotypes (Supplementary Table 2). A phylogenic tree without the outgroups is also included in Supplementary Fig. 18. Monophyletic clades and tree branches remained similar.

\section{Assessment of the Microcystis genomic (within-colonies) variation versus intra-genotype variation (between colonies)}

We first confirmed that Microcystis is haploid, as polyploidy has been observed among other cyanobacteria [82]. We estimated ploidy variation in Microcystis colonies using k-mer frequencies and raw sequences. We first mapped the reads of each colony (containing reads from both Microcystis and its microbiome) to a Microcystis reference genome using BBmap with minimum nucleotide identity of 99\% [83]. Mapped reads were extracted using Picard (http://broadinstitute.github.io/picard/) and analyzed using Genomescope and Smudgeplot (https://github. com/tbenavi1/genomescope2.0; https://github.com/ KamilSJaron/ smudgeplot). All colonies appeared to be haploid, with a low rate of heterozygosity that could be due to paralogs.

To determine whether Microcystis colonies were likely formed by clonal cell division or cell aggregation, we called SNVs within colonies and between colonies of the same genotype. As a point of comparison, we also called SNVs that occurred over a period of approximately 6 years in nineteen laboratory cultures of Microcystis with genome sequences reported previously [31]. We used snippy (v4.4.0) (https://github.com/ tseemann/snippy) with default parameters to call SNVs. Genotypes represented by only one sampled colony were excluded from the analysis (G02, G04, G09, G11, G12, G16 and G18).

SNV calling within and between colonies was executed by mapping reads against reference genomes. This was done independently for each genotype. We selected at least four reference genomes per genotype when possible. SNVs within colonies were detected by mapping the reads of the references to their respective genome assemblies. SNVs between colonies were detected by mapping the reads of different colonies of the same genotype to the genome assemblies of the references. We ignored positions where the reference nucleotide was poorly supported (threshold percentage for the minor variant $<14.4 \%$; mean $=1.1 \%)$ by the reads in both the within- and between-colony read mapping analyses because these were considered to be assembly errors.

\section{Identifying associated bacterial genomes in colonies}

Non-Microcystis MAGs from each colony were classified in 72 species based on taxonomical analysis and ANI values $\geq 96 \%$. Because individual assemblies could affect MAG completeness, we created a custom database of the $59 \mathrm{AB}$ genomes from Quebec, and another database for the 18 species from Brazil. Using MIDAS (v1.3.0) [39], we mapped the reads from each colony (downsampled to $8,000,000$ reads per colony) against the custom databases to estimate the relative abundance and coverage for each of the 72 AB species. These custom databases contain one representative genome per species, chosen as the highest quality MAG within that species. We defined a species to be present when it had a genome-wide average depth of coverage of $1 \times$ or more. This allowed us to generate a matrix of $A B$ presence or absence across colonies. To take into account both assembled and unassembled taxa, the microbiome composition was assessed using two additional approaches: (1) by mapping the reads against the complete MIDAS reference genome database containing 5952 species and (2) by using the read counts and taxonomical annotation based on the program Kaiju (v1.8) with default parameters [84].

\section{Microcystis' microbiome composition variation according to environmental variables and host genotype}

The following statistical analyses were conducted in $\mathrm{R}$ (v3.6.2). We first performed a distance-based RDA using the capscale function from the $\mathrm{R}$ vegan package 
(v2.5.61, [85]) with the square root of the Bray-Curtis distance from a coverage table describing the composition of the Microcystis microbiome for each genotype. The variables included genotype information, presence/absence of $m c y$ genes, temperature, $\mathrm{pH}$, site (Canada or Brazil) and the temporal variables years and months. In a second approach, we calculated the beta diversity using the same dissimilarity distance and tested the differences in Microcystis microbiome structure variation using permutational multivariate analysis of variance (PERMANOVA, [86]) with the adonis() function from the $\mathrm{R}$ vegan package. As PERMANOVA tests can be sensitive to dispersion, we have also tested for dispersion in the data by performing an analysis of multivariate homogeneity (PERMDISP, [87]) with the permuted betadisper() function of the vegan package).

We quantified phylosymbiosis by comparing the phylogenetic distance matrix of Microcystis genotypes and the microbiome composition distance matrix using a Mantel test (999 permutations, Spearman correlation) and the protest() $\mathrm{R}$ function to test the non-randomness between these two matrices (999 permutations) ( $\mathrm{R}$ vegan package). The pairwise phylogenetic distance matrix was estimated using the RAxML tree of the Microcystis core genome and the cophenetic.phylo function of the ape $\mathrm{R}$ package (v5.3) [88]. The results based on the extended MIDAS reference database and Kaiju taxonomic assignments are described in the supplementary material (Supplementary Table 8).

\section{Microcystis genotypic diversity from metagenomic samples}

Microcystis genomes from Quebec and Brazil were classified into 14 and four genotypes, respectively. This genotype classification was based on pairwise genome similarities greater or equal to $99 \%$. Using the Microcystis genotypes and the software MIDAS (v1.3.0) [39], we built two custom gene marker databases for the Microcystis genotypes (15 universal single-copy gene families), one for genotypes from Quebec and the other for genotypes from Brazil.

Using MIDAS and the custom databases, we estimated the relative abundances, the read counts and the read coverage of the Microcystis genotypes in 72 shotgun metagenomes from Lake Champlain, Quebec (62 metagenomes from a long-term experiment (2006 to 2016, excluding 2007 and 2014), plus 10 metagenomes from 2017 and 2018). Due the low number of Microcystis genotypes and metagenomes (6 sampling points for Brazil during 2018) from Brazil, these samples were not formally analyzed. Metagenomic reads with similarity greater or equal to $99 \%$ were mapped against the MIDAS database of Microcystis genotypes. We used 14,000,000 reads per metagenome after downsampling to the lowest-coverage metagenome (Supplementary Table 3). The metagenome sequencing from Brazil were mapped against a separate MIDAS database of the four Microcystis genotypes from Brazil (Supplementary Fig. 19).

To test if the 14 Microcystis genotypes identified in the colony genomes are representative of the diversity present in the Lake Champlain metagenomes, we first mapped the downsampled metagenomic reads to a custom database including a single reference Microcystis genome (M083S1) (alignment identity cutoff = 96\%), and also mapped the reads to the database including all the 14 genotypes (alignment identity cutoff $=99 \%$ ). By using a cutoff value equal to $96 \%$, we expect to recover most sequences from the Microcystis genus, regardless of which genotype the reads come from. We recovered 102,608 reads at 99\% identity and 109,729 at $96 \%$, showing that the 14 genotypes (defined at 99\% identity) account for $93.5 \%$ of the Microcystis reads in the metagenome samples. Additionally, we observed that the total coverage using all the Microcystis genotypes (alignment identity cutoff = 99\%) and the total coverage using a single Microcystis genome as a reference (alignment identity cutoff = 96\%) are nearly perfectly correlated (correlation coefficient $r=1, P<2.2 \mathrm{e}-16)$ (Spearman correlation) (Supplementary Fig. 20).

\section{Microcystis genotypic diversity variation according to environmental variables}

To determine the factors that explain the variation in Microcystis community composition, we used a dataset of 42 metagenomes and 14 genotypes from Lake Champlain. Metagenomes with incomplete metadata were excluded. We focused on Lake Champlain as we observed a greater diversity of Microcystis genotypes compared to Brazil, including both microcystinproducing and non-producing genotypes. Statistical analyses were performed in $\mathrm{R}$ (v3.6.2). We first used dbRDA with the square root of the Bray-Curtis distance matrix to investigate Microcystis-environment relationships $[89,90]$ (capscale function from vegan $R$ package, $R$ vegan package). Variables were preselected using the ordiR2step $\mathrm{R}$ function ( $\mathrm{R}$ vegan package) (see Supplementary Methods). The environmental matrix variables included: total phosphorus in micrograms per litre $(\mu \mathrm{g} / \mathrm{L})(\mathrm{TP})$, total nitrogen in $\mu \mathrm{g} /$ $\mathrm{L}(\mathrm{TN})$, soluble reactive phosphorus in $\mu \mathrm{g} / \mathrm{L}$ (DP), dissolved nitrogen in $\mu \mathrm{g} / \mathrm{L}$ (DN), 1-week-cumulative precipitation in millimetres $(\mathrm{mm}), 1$-week-average air temperature in Celsius, temporal variables (Years, Months and Season) and sampling sites within Lake Champlain (Pelagic or Littoral) (Supplementary Table 3) [61]. To determine the significance of constraints, 
we used the anova.cca() function from the $\mathrm{R}$ vegan package.

We also calculated the beta diversity between groups of samples using the Phyloseq R package (v1.30.0) [91] and the square root of Bray-Curtis distance. We used nonmetric multi-dimensional scaling (NMDS, from the phyloseq package that incorporates the metaMDS() function from the $\mathrm{R}$ vegan $[85,92,93]$ package to ordinate the data. Differences in community structure between groups were tested using permutational multivariate analysis of variance (PERMANOVA, [86]) with the adonis() function. As described previously for similar analysis, we also tested for differences in dispersion between groups (genotypes) with the permuted betadisper() function.

\section{Correlations between and Microcystis and its microbiome in freshwater metagenomes from Canada}

Using the 59 species identified in the Microcystis microbiome from Canada and the software MIDAS, we built a custom gene marker database of 15 universal single-copy gene families. This database also included a reference genome from Microcystis (M083S1) and two Dolichospermum reference genomes $(D$. circinale AWQC131C and AWQC310F). Using MIDAS, we estimated the relative abundances, reads count, and the read coverage of each $\mathrm{AB}$ species in 72 shotgun metagenomes from Quebec, Canada. Reads were mapped against the custom database including the $\mathrm{AB}$ species. A cuff-off value of nucleotide identity greater or equal to $96 \%$ was used for the read mapping. By merging the values (coverage and read counts) for species within the same genus, obtained coverage and read counts at the genus level, for 32 genera of AB. We used the Spearman correlation to investigate patterns of co-occurrence between Microcystis, Dolichospermum and the $\mathrm{AB}$ species and genera in environmental metagenomes. First, the read counts in the matrices containing the genera and species were used to estimate the Spearman correlation values $(r)$ and $P$ values between pair of species or genera by using the rcorr() function of the Hmisc (v4.3.0) R package [94]. We also calculated Spearman correlations on the coverage values, yielding similar results. $P$ values were corrected to control the false discovery rate using the qvalue() function from the qvalue (v2.18.0) R package. We also estimated the correlation between Microcystis and the $\mathrm{AB}$ using the software FastSpar (v0.0.10) [95]. This method is a faster implementation of the Sparse Correlation for Compositional Data algorithm (SparCC) [96]. The significance of the test was evaluated using 100 permutations and a bootstrap of 1000. In general, the most prevalent $\mathrm{AB}$ taxa in Microcystis colonies had significant correlation $(P<0.05)$ with Microcystis using both Spearman and SparCC.

\section{Co-phylogeny between Microcystis and members of the microbiome}

The nine most prevalent $A B$ genera were selected for co-phylogeny analysis, which would be underpowered to detect phylogenetic associations with low-prevalence bacteria (i.e., small phylogenies). Core genomes were generated using panX and core alignments were computed as described above, for each $\mathrm{AB}$ genus. Phylogenic core genome trees were built individually for each genus using RAxML under the GTRGAMMA substitution model [78]. Support values of the tree nodes were estimated using 100 bootstrap replicates. Patristic distances (pairwise distances between pairs of tips on a tree) for the Microcystis and $A B$ phylogenies were estimated using the cophenetic.phylo() function from the ape R-package [88]. The Microcystis core genome tree and the tree of the $A B$ were compared using Parafit test (parafit() function of the ape $\mathrm{R}$ package) (see Supplementary Methods) [88, 97]. Cophylogeny trees were built using the function cophylo() from the phytools R package [98].

\section{Recent HGT between Microcystis and associated bacteria}

To infer recent HGT events between Microcystis and $A B$, we first inferred the pangenomes for each combination of one $\mathrm{AB}$ and Microcystis, and repeated this for the $72 \mathrm{AB}$ species. Core and accessory genes with a minimum percentage identity for blastp equal to $99 \%$ were identified. We retained those clusters of genes present in at least four genomes, and present in both $A B$ and Microcystis. The remaining putatively horizontal transferred genes were annotated in 23 COG (clusters of orthologous groups) categories using eggNOG-mapper (v2.0.1) [99]. Using the package STAMP (v2.1.3) and a chi-squared test, we estimated if there were statistical differences in the COG categories between Microcystis core genes and the putative horizontally transferred genes [100]. $P$ values were corrected using the Benjamini-Hochberg (controlling the false discovery rate) method. We also estimated HGT events between Microcystis and associated species using a second method, Metachip (v1.8.2) (default parameters). The Metachip approach uses both the best match approach (blastn) and a phylogenetic approach to infer HGT (reconciliation between a gene tree and its species tree) [41].

\section{Gene functional annotation}

The Microcystis and AB genomes were functionally annotated using enrichM (v0.5.0) (https://github.com/ geronimp/enrichM) [101]. A PCA based on the presence/ absence of KEGG Orthologous genes (KO) in Microcystis and $\mathrm{AB}$ genera was generated using the option 'enrichment' in enrichM. Genome groups (Microcystis vs each $\mathrm{AB}$ genus) were compared using the same option. KEGG 
modules differentially abundant in Microcystis or the $\mathrm{AB}$ genus were filtered based on a completeness greater or equal to $70 \%$. Additionally, we used the program gapseq (v1.1) with default parameters and the MetaCyc database $[43,45]$ to validate the presence the metabolic pathways involved in the biosynthesis of biotin, cobalamin, thiamine, carotenoid and nitrogen fixation.

Microcystis and AB genomes (109 Microcystis and 391 associated genomes) were annotated using Roary (v3.13.0) [102]. The resulting genomes in GenBank format were used to predict the biosynthetic gene clusters (BGCs) using default parameters (--taxon bacteria --cb-general --cbknownclusters --cb-subclusters --asf --pfam2go --smcogtrees --genefinding-tool prodigal-m) in antiSMASH (v5.1.2) $[42,103]$. The BIG-SCAPE package (v1.0.1) with default parameters analyzed the ANTISMASH BGCs and based on a similarity network classified them into Gene Cluster Families (GCFs) [104]. BGCs were classified in BiG-SCAPE classes (e.g., polyketide synthases nonribosomal peptide synthetases (NRPSs), post-translationally modified peptides (RiPPs) and terpenes. A total of 2395 BGCs were identified in 415 genomes.

\section{Metabolic pathway reconstruction}

We reconstructed genome scale metabolic models from the genomic sequences of one Microcystis genotype (G01) and its microbiome composed of Roseomonas, Rhodobacter, Methylobacterium, Phenylobacterium and Pseudanabaena, which are among the most prevalent AB for Microcystis as a whole. We used MAG annotations from prokka (v1.14.0) [73] as input for CarveMe (v1.5.0) [105] to generate the models, with default parameters. We next assessed the metabolic interactions using SMETANA (v1.1.0) [46] excluding inorganic compounds which we assumed are generally present in the water system, and using the -molweight parameter to minimize the total mass of the compounds in the media, which is less prone to degenerate solutions. SMETANA is a flux balance analysis-based simulation tool that first estimates the minimal amount of nutrients required for the community to survive. A "detailed" analysis estimates the interspecies dependencies under a minimal constrained nutritional environment, by calculating a species coupling score (SCS), metabolite uptake score (MUS), metabolite production score (MTS) and a SMETANA score (a combination of the three other scores). A second "global" analysis characterizes the general interactions of all members in noninteracting and interacting communities by calculating the metabolic resource overlap (MRO) and metabolic interaction potential (MIP) [46]. As a control, we analyzed the interactions with taxa (i.e., Phycisphaerales, Burkholderia and Acidobacteriaceae) that were not co-occurrent with Microcystis in metagenomes based on SparCC and Spearman correlation results. The output was empty meaning that there were no cross-feeding interactions between Microcystis and these taxa. When at least one species depends on the others to survive, cross-feeding interactions are listed in the SMETANA detailed output file.

\section{Supplementary Information}

The online version contains supplementary material available at https://doi. org/10.1186/s40168-021-01140-8.

Additional file 1. Supplementary methods. DNA extraction colonies.

Additional file 2. Supplementary methods. dbRDA phylosymbiosis ( $R$ script).

Additional file 3. Supplementary methods. Co-phylogeny (R script).

Additional file 4. Supplementary figures.

Additional file 5: Table S1. Microcystis genome characteristics.

Additional file 6: Table S2. ANIm and ANIb values.

Additional file 7: Table S3. Metadata for metagenomic samples from Lake Champlain and Pampulha Reservoir.

Additional file 8: Table S4. SNVs within and between Microcystis colonies. SNVs accumulated in 19 Microcystis laboratory cultures after $\sim 6$ years of culture are also included.

Additional file 9: Table S5. $A B$ genome characteristics.

Additional file 10: Table S6. Kaiju core species according to various read counts cut-off.

Additional file 11: Table S7. $d b R D A$ and PERMANOVA results.

Additional file 12: Table S8. (A) PERMANOVA and (B) Phylosymbiosis results both based on the microbial community composition that includes assembled and non-assembled taxa.

Additional file 13: Table S9. Single-copy taxonomic marker genes for MIDAS analysis.

Additional file 14: Table S10. Spearman and SparCC correlations. Additional file 15: Table S11. Gene families involved in HGT using Metachip. The table shows the genes identified with Metachip, and 31 genes families that were also identified with an additional method.

Additional file 16: Table S12. Presence/absence of certain metabolic pathways involved in the biosynthesis of biotin, cobalamin, thiamin and carotenoid, and nitrogen assimilation.

Additional file 17: Table S13. KEGG modules present in $A B$.

Additional file 18: Table S14. Metabolic exchange analysis. (A) SMETANA output from a modeled community of Microcystis genotype G01 (M007S1) and the five members of its microbiome in minimal media. (B) Pairwise SMETANA analysis of Microcystis and five members of its microbiome in complete media. (C)Negative control pairwise SMETANA analysis of Microcystis and three non-associated bacteria in complete media.

Additional file 19: Table S15. Colonies sequenced per each sampling time point.

Additional file 20: Table S16. Read quality scores for the Microcystis colonies and the environmental metagenomes. Quality scores for the colonies ( $A$ and $B$ ) and for the lake metagenomes ( $C$ and $D)$.

Additional file 21: Table S17. ANIm between AB genomes.

Additional file 22: Table S18. Taxonomical classification of contigs in uncomplete MAGs based on CAT analysis.

Additional file 23: Table S19. Reference genomes.

\section{Acknowledgements}

We are grateful to Julie Marleau, Miria Elias and Alberto Mazza for assistance in the sampling. We also thank Seth Bordenstein for suggesting the term "microbe-by-microbiome phylosymbiosis" to describe our results. 


\section{Authors' contributions}

BJS, NT and OMPC designed the study. OMPC, NT, AG, LCBM.and NF performed the lab experiments. NT and OMPC performed the data analyses. EM and OMPC performed the cophylogeny. BJS, NT and OMPC wrote the manuscript. BJS, NT, OMPC, AG, YT and NF contributed to its reviewing and editing. The author(s) read and approved the final manuscript.

\section{Funding}

This work was supported by the Genome Québec and Genome Canadafunded ATRAPP Project (Algal blooms, Treatment, Risk Assessment, Prediction and Prevention). Colonies and water samples from Brazil were obtained thanks to a FAPEMIG grant to AG. We also want to acknowledge the financial support of the National Research Council.

\section{Availability of data and materials}

Raw sequences and metagenome-assembled genomes (MAGs) are available in NCBI under Bioproject numbers PRJNA507251 and PRJNA662092.

\section{Declarations}

Ethics approval and consent to participate

Not applicable.

\section{Consent for publication}

Not applicable.

\section{Competing interests}

The authors declare that they have no conflicts of interest.

\section{Author details}

'Département de Sciences Biologiques, Université de Montréal, Montréal, Québec, Canada. ${ }^{2}$ Federal University of Minas Gerais, Belo Horizonte, Minas Gerais, Brazil. ${ }^{3}$ National Research Council of Canada, Montreal, Québec, Canada. ${ }^{4}$ Department of Microbiology \& Immunology, McGill University, Montreal, Québec, Canada. ${ }^{5}$ McGill Genome Centre, McGill University, Montreal, Québec, Canada.

Received: 26 November 2020 Accepted: 2 August 2021

Published online: 27 September 2021

\section{References}

1. Levesque B, Gervais MC, Chevalier P, Gauvin D, Anassour-Laouan-Sidi E, Gingras $S$, et al. Prospective study of acute health effects in relation to exposure to cyanobacteria. Sci Total Environ. 2014;466-467:397-403.

2. Bell W, Mitchell R. Chemotactic and growth responses of marine bacteria to algal extracellular products. Biol Bull. 1972;143(2):265-77. https://doi.org/1 $0.2307 / 1540052$.

3. Seymour JR, Amin SA, Raina JB, Stocker R. Zooming in on the phycosphere: the ecological interface for phytoplankton-bacteria relationships. Nat Microbiol. 2017;2(7):17065. https://doi.org/10.1038/nmicrobiol.2017.65.

4. Amin SA, Parker MS, Armbrust EV. Interactions between diatoms and bacteria. Microbiol Mol Biol Rev. 2012;76(3):667-84. https://doi.org/10.1128/ MMBR.00007-12

5. Paerl HW. Microscale physiological and ecological studies of aquatic cyanobacteria: macroscale implications. Microsc Res Tech. 1996;33(1):4772. https://doi.org/10.1002/(SICI)1097-0029(199601)33:1<47:.AID-JEMT6>3. $0 . \mathrm{CO} ; 2-\mathrm{Y}$.

6. Cho DH, Ramanan R, Heo J, Lee J, Kim BH, Oh HM, et al. Enhancing microalgal biomass productivity by engineering a microalgal-bacterial community. Bioresour Technol. 2015;175:578-85. https://doi.org/10.1016/j. biortech.2014.10.159.

7. Amin SA, Hmelo LR, van Tol HM, Durham BP, Carlson LT, Heal KR, et al. Interaction and signalling between a cosmopolitan phytoplankton and associated bacteria. Nature. 2015;522(7554):98-101. https://doi.org/10.1038/ nature14488.

8. Van Mooy BA, Hmelo LR, Sofen LE, Campagna SR, May AL, Dyhrman ST, et al. Quorum sensing control of phosphorus acquisition in Trichodesmium consortia. ISME J. 2012;6(2):422-9. https://doi.org/10.1038/ismej.2011.115.

9. Frischkorn KR, Rouco M, Van Mooy BAS, Dyhrman ST. Epibionts dominate metabolic functional potential of Trichodesmium colonies from the oligotrophic ocean. ISME J. 2017;11(9):2090-101. https://doi.org/10.1038/ ismej.2017.74.

10. Paerl HW. Growth and reproductive strategies of freshwater blue-green algae (Cyanobacteria), Growth and reproductive strategies of freshwater phytoplankton; 1988. p. 261-315.

11. Worm J, Sondergaard M. Dynamics of heterotrophic bacteria attached to Microcystis spp. (Cyanobacteria). Aquat Microb Ecol. 1998;14:19-28. https:// doi.org/10.3354/ame014019.

12. Brunberg AK. Contribution of bacteria in the mucilage of Microcystis spp. (Cyanobacteria) to benthic and pelagic bacterial production in a hypereutrophic lake. Fems Microbiol Ecol. 1999;29(1):13-22. https://doi.org/1 0.1111/j.1574-6941.1999.tb00594.x.

13. Parveen B, Ravet V, Djediat C, Mary I, Quiblier C, Debroas D, et al. Bacterial communities associated with Microcystis colonies differ from free-living communities living in the same ecosystem. Environ Microbiol Rep. 2013;5: $716-24$

14. Jankowiak JG, Gobler CJ. The composition and function of microbiomes within Microcystis colonies are significantly different than native bacterial assemblages in two North American lakes. Front Microbiol. 2020;11. https:// doi.org/10.3389/fmicb.2020.01016.

15. Dziallas C, Grossart HP. Temperature and biotic factors influence bacterial communities associated with the cyanobacterium Microcystis sp. Environ Microbiol. 2011;13(6):1632-41. https://doi.org/10.1111/j.1462-2 920.2011.02479.x

16. Cook KV, Li C, Cai H, Krumholz LR, Hambright KD, Paerl HW, et al. The global Microcystis interactome. Limnol Oceanogr. 2020;65(Suppl 1):S194-207. https://doi.org/10.1002/Ino.11361

17. Shia LM, Cai YF, Wang XY, Li PF, Yu Y, Kong FX. Community structure of bacteria associated with Microcystis colonies from cyanobacterial blooms. J Freshwater Ecol. 2010;25(2):193-203. https://doi.org/10.1080/02705060.2010. 9665068 .

18. Berg KA, Lyra C, Sivonen K, Paulin L, Suomalainen S, Tuomi P, et al. High diversity of cultivable heterotrophic bacteria in association with cyanobacterial water blooms. ISME J. 2009;3(3):314-25. https://doi.org/10.1 038/ismej.2008.110.

19. Shen H, Niu Y, Xie P, Tao M, Yang X. Morphological and physiological changes in Microcystis aeruginosa as a result of interactions with heterotrophic bacteria. Freshwater Biol. 2011;56(6):1065-80. https://doi.org/1 0.1111/j.1365-2427.2010.02551.x.

20. Wang WJ, Shen H, Shi PL, Chen J, Ni LY, Xie P. Experimental evidence for the role of heterotrophic bacteria in the formation of Microcystis colonies. J Appl Phycol. 2016;28(2):1111-23. https://doi.org/10.1007/s10811-015-0659-5.

21. Xiao M, Willis A, Burford MA, Li M. Review: a meta-analysis comparing celldivision and cell-adhesion in Microcystis colony formation. Harmful Algae. 2017;67:85-91. https://doi.org/10.1016/j.hal.2017.06.007.

22. Wang X, Qin B, Gao G, Paerl HW. Nutrient enrichment and selective predation by zooplankton promote Microcystis (Cyanobacteria) bloom formation. J Plankton Res. 2010;32(4):457-70. https://doi.org/10.1093/plankt/fbp143.

23. Kim M, Shin B, Lee J, Park HY, Park W. Culture-independent and culturedependent analyses of the bacterial community in the phycosphere of cyanobloom-forming Microcystis aeruginosa. Sci Rep. 2019;9(1):20416. https://doi.org/10.1038/s41598-019-56882-1.

24. Lim SJ, Bordenstein SR. An introduction to phylosymbiosis. Proc Biol Sci. 2020;287:20192900.

25. Groussin M, Mazel F, Sanders JG, Smillie CS, Lavergne S, Thuiller W, et al. Unraveling the processes shaping mammalian gut microbiomes over evolutionary time. Nat Commun. 2017;8(1):14319. https://doi.org/10.1038/ ncomms14319.

26. Yeoh YK, Dennis PG, Paungfoo-Lonhienne C, Weber L, Brackin R, Ragan MA, et al. Evolutionary conservation of a core root microbiome across plant phyla along a tropical soil chronosequence. Nat Commun. 2017;8(1):215 https://doi.org/10.1038/s41467-017-00262-8.

27. Pollock FJ, McMinds R, Smith S, Bourne DG, Willis BL, Medina M, et al. Coralassociated bacteria demonstrate phylosymbiosis and cophylogeny. Nat Commun. 2018;9(1):4921. https://doi.org/10.1038/s41467-018-07275-x.

28. Mazel F, Davis KM, Loudon A, Kwong WK, Groussin M, Parfrey LW. Is host filtering the main driver of phylosymbiosis across the tree of life? mSystems. 2018:3:e00097-18.

29. Groussin M, Mazel F, Alm EJ. Co-evolution and co-speciation of host-gut bacteria systems. Cell Host Microbe. 2020;28(1):12-22. https://doi.org/10.101 6/j.chom.2020.06.013. 
30. Harke MJ, Steffen MM, Gobler CJ, Otten TG, Wilhelm SW, Wood SA, et al. A review of the global ecology, genomics, and biogeography of the toxic cyanobacterium, Microcystis spp. Harmful Algae. 2016;54:4-20. https://doi. org/10.1016/j.hal.2015.12.007.

31. Perez-Carrascal OM, Terrat Y, Giani A, Fortin N, Greer CW, Tromas N, et al. Coherence of Microcystis species revealed through population genomics. ISME J. 2019;13(12):2887-900. https://doi.org/10.1038/s41396-019-0481-1.

32. Jackrel SL, White JD, Evans JT, Buffin K, Hayden K, Sarnelle O, et al. Genome evolution and host-microbiome shifts correspond with intraspecific niche divergence within harmful algal bloom-forming Microcystis aeruginosa. Mol Ecol. 2019;28(17):3994-4011. https://doi.org/10.1111/mec.15198.

33. Campbell JH, O'Donoghue P, Campbell AG, Schwientek P, Sczyrba A, Woyke T, et al. UGA is an additional glycine codon in uncultured SR1 bacteria from the human microbiota. Proc Natl Acad Sci. 2013;110(14):5540-5. https://doi. org/10.1073/pnas.1303090110.

34. Wilson AE, Sarnelle O, Neilan BA, Salmon TP, Gehringer MM, Hay ME. Genetic variation of the bloom-forming cyanobacterium Microcystis aeruginosa within and among lakes: implications for harmful algal blooms. Appl Environ Microbiol. 2005;71(10):6126-33. https://doi.org/10.1128/AEM. 71.10.6126-6133.2005.

35. Dittmann E, Neilan BA, Erhard M, von Dohren H, Borner T. Insertional mutagenesis of a peptide synthetase gene that is responsible for hepatotoxin production in the cyanobacterium Microcystis aeruginosa PCC 7806. Mol Microbiol. 1997;26(4):779-87. https://doi.org/10.1046/j.1365-2958.1 997.6131982.x

36. Peres-Neto PR, Jackson DA. How well do multivariate data sets match? The advantages of a Procrustean superimposition approach over the Mantel test. Oecologia. 2001;129(2):169-78. https://doi.org/10.1007/s004420100720.

37. Kurmayer R, Dittmann E, Fastner J, Chorus I. Diversity of microcystin genes within a population of the toxic cyanobacterium Microcystis spp. in Lake Wannsee (Berlin, Germany). Microb Ecol. 2002;43(1):107-18. https://doi.org/1 0.1007/s00248-001-0039-3.

38. Briand E, Escoffier N, Straub C, Sabart M, Quiblier C, Humbert J-F. Spatiotemporal changes in the genetic diversity of a bloom-forming Microcystis aeruginosa (Cyanobacteria) population. ISME J. 2009;3(4):419-29. https://doi.org/10.1038/ismej.2008.121

39. Nayfach S, Rodriguez-Mueller B, Garud N, Pollard KS. An integrated metagenomics pipeline for strain profiling reveals novel patterns of bacterial transmission and biogeography. Genome Res. 2016;26(11):1612-25. https://doi.org/10.1101/gr.201863.115.

40. Smillie CS, Smith MB, Friedman J, Cordero OX, David LA, Alm EJ. Ecology drives a global network of gene exchange connecting the human microbiome. Nature. 2011;480(7376):241-4. https://doi.org/10.103 8/nature10571.

41. Song W, Wemheuer B, Zhang S, Steensen $\mathrm{K}$, Thomas T. MetaCHIP: community-level horizontal gene transfer identification through the combination of best-match and phylogenetic approaches. Microbiome. 2019;7(1):36. https://doi.org/10.1186/s40168-019-0649-y.

42. Blin K, Shaw S, Steinke K, Villebro R, Ziemert N, Lee SY, et al. antiSMASH 5.0: updates to the secondary metabolite genome mining pipeline. Nucleic Acids Res. 2019;47(W1):W81-W7. https://doi.org/10.1093/nar/gkz310.

43. Zimmermann J, Kaleta C, Waschina S. gapseq: informed prediction of bacterial metabolic pathways and reconstruction of accurate metabolic models. Genome Biol. 2021;22:81.

44. Paerl HW, Tucker J, Bland PT. Carotenoid enhancement and its role in maintaining blue-green algal (Microcystis aeruginosa) surface blooms1. Limnol Oceanogr. 1983;28(5):847-57. https://doi.org/10.4319/lo.1983.28.5. 0847.

45. Caspi R, Billington R, Keseler IM, Kothari A, Krummenacker M, Midford PE, et al. The MetaCyc database of metabolic pathways and enzymes - a 2019 update. Nucleic Acids Res. 2020;48(D1):D445-D53. https://doi.org/10.1093/na r/gkz862.

46. Zelezniak A, Andrejev S, Ponomarova O, Mende DR, Bork P, Patil KR. Metabolic dependencies drive species co-occurrence in diverse microbial communities. Proc Natl Acad Sci U S A. 2015;112(20):6449-54. https://doi. org/10.1073/pnas.1421834112.

47. Gerloff GC, Fitzgerald GP, Skoog F. The mineral nutrition of Microcystis aeruginosa. Am J Bot. 1952;39(1):26-32. https://doi.org/10.1002/j.1537-2197.1 952.tb13041.x

48. Tromas N, Taranu ZE, Martin BD, Willis A, Fortin N, Greer CW, et al. Niche separation increases with genetic distance among bloom-forming cyanobacteria. Front Microbiol. 2018;9:438. https://doi.org/10.3389/fmicb.201 8.00438.

49. Chun SJ, Cui YS, Lee CS, Cho AR, Baek K, Choi A, et al. Characterization of distinct cyanoHABs-related modules in microbial recurrent association network. Front Microbiol. 2019;10. https://doi.org/10.3389/fmicb.2019.01637.

50. Zhang Z, Fan X, Peijnenburg WJGM, Zhang M, Sun L, Zhai Y, et al. Alteration of dominant cyanobacteria in different bloom periods caused by abiotic factors and species interactions. J Environ Sci. 2021;99:1-9. https://doi.org/1 0.1016/j.jes.2020.06.001.

51. Xiao M, Li M, Reynolds CS. Colony formation in the cyanobacterium Microcystis. Biol Rev. 2018;93(3):1399-420. https://doi.org/10.1111/brv.12401.

52. Yang Z, Kong F, Shi X, Zhang M, Xing P, Cao H. Changes in the morphology and polysaccharide content of Microcystis aeruginosa (Cyanobacteria) during flagellate grazing. J Phycol. 2008;44(3):716-20. https://doi.org/10.1111/j.15298817.2008.00502.x

53. Li Q, Lin F, Yang C, Wang J, Lin Y, Shen M, et al. A large-scale comparative metagenomic study reveals the functional interactions in six bloom-forming Microcystis-epibiont communities. Front Microbiol. 2018;9:746. https://doi. org/10.3389/fmicb.2018.00746.

54. Douglas AE, Werren JH. Holes in the hologenome: why host-microbe symbioses are not holobionts. mBio. 2016;7:e02099.

55. Kosourov S, Murukesan G, Jokela J, Allahverdiyeva Y. Carotenoid biosynthesis in Calothrix sp. 336/3: composition of carotenoids on full medium, during diazotrophic growth and after long-term H2 photoproduction. Plant Cell Physiol. 2016;57(11):2269-82. https://doi.org/10.1093/pcp/pcw143.

56. Pattanaik B, Lindberg P. Terpenoids and their biosynthesis in cyanobacteria. Life. 2015;5(1):269-93. https://doi.org/10.3390/life5010269.

57. Dieser M, Greenwood M, Foreman CM. Carotenoid pigmentation in antarctic heterotrophic bacteria as a strategy to withstand environmental stresses. Arctic Antarctic Alpine Res. 2010;42(4):396-405. https://doi.org/10.1 657/1938-4246-42.4.396.

58. Beiko RG, Harlow TJ, Ragan MA. Highways of gene sharing in prokaryotes. Proc Natl Acad Sci USA. 2005;102(40):14332-7. https://doi.org/10.1073/pnas. 0504068102.

59. Morris JJ, Lenski RE, Zinser ER. The Black Queen Hypothesis: evolution of dependencies through adaptive gene loss. mBio. 2012;3:e00036-12.

60. Schmidt KC, Jackrel SL, Smith DJ, Dick GJ, Denef VJ. Genotype and host microbiome alter competitive interactions between Microcystis aeruginosa and Chlorella sorokiniana. Harmful Algae. 2020;99:101939. https://doi.org/1 $0.1016 / j . h a l .2020 .101939$.

61. Tromas N, Fortin N, Bedrani L, Terrat Y, Cardoso P, Bird D, et al. Characterising and predicting cyanobacterial blooms in an 8-year amplicon sequencing time course. ISME J. 2017;11(8):1746-63. https://doi.org/10.1038/ismej.2017.58.

62. Chen S, Zhou Y, Chen Y, Gu J. fastp: an ultra-fast all-in-one FASTQ preprocessor. Bioinformatics. 2018;34(17):i884-i90. https://doi.org/10.1093/ bioinformatics/bty560.

63. Bolger AM, Lohse M, Usadel B. Trimmomatic: a flexible trimmer for Illumina sequence data. Bioinformatics. 2014;30(15):2114-20. https://doi.org/10.1093/ bioinformatics/btu170.

64. Li D, Luo R, Liu CM, Leung CM, Ting HF, Sadakane K, et al. MEGAHIT v1.0: a fast and scalable metagenome assembler driven by advanced methodologies and community practices. Methods. 2016;102:3-11.

65. Eren AM, Esen OC, Quince C, Vineis JH, Morrison HG, Sogin ML, et al. Anvi'o: an advanced analysis and visualization platform for 'omics data. PeerJ. 2015; 3:e1319. https://doi.org/10.7717/peerj.1319.

66. Trembath-Reichert E, Butterfield DA, Huber JA. Active subseafloor microbial communities from Mariana back-arc venting fluids share metabolic strategies across different thermal niches and taxa. ISME J. 2019;13(9):226479. https://doi.org/10.1038/s41396-019-0431-y.

67. Love CR, Arrington EC, Gosselin KM, Reddy CM, BAS VM, Nelson RK, et al. Microbial production and consumption of hydrocarbons in the global ocean. Nat Microbiol. 2021;6(4):489-98.

68. Watson AR, Füssel J, Veseli I, DeLongchamp JZ, Silva M, Trigodet F, et al. Adaptive ecological processes and metabolic independence drive microbial colonization and resilience in the human gut. bioRxiv. 2021. https://doi. org/10.1101/2021.03.02.433653.

69. Langmead B, Salzberg SL. Fast gapped-read alignment with Bowtie 2. Nat Methods. 2012;9(4):357-U54. https://doi.org/10.1038/nmeth.1923.

70. Kim D, Song L, Breitwieser FP, Salzberg SL. Centrifuge: rapid and sensitive classification of metagenomic sequences. Genome Res. 2016;26(12):1721-9. https://doi.org/10.1101/gr.210641.116. 
71. Lee STM, Kahn SA, Delmont TO, Shaiber A, Esen OC, Hubert NA, et al. Tracking microbial colonization in fecal microbiota transplantation experiments via genome-resolved metagenomics. Microbiome. 2017;5(1):50. https://doi.org/10.1186/s40168-017-0270-x.

72. Olm MR, Brown CT, Brooks B, Banfield JF. dRep: a tool for fast and accurate genomic comparisons that enables improved genome recovery from metagenomes through de-replication. ISME J. 2017;11(12):2864-8. https:// doi.org/10.1038/ismej.2017.126.

73. Seemann T. Prokka: rapid prokaryotic genome annotation. Bioinformatics. 2014;30(14):2068-9. https://doi.org/10.1093/bioinformatics/btu153.

74. Jain C, Rodriguez RL, Phillippy AM, Konstantinidis KT, Aluru S. High throughput ANI analysis of $90 \mathrm{~K}$ prokaryotic genomes reveals clear species boundaries. Nat Commun. 2018;9(1):5114. https://doi.org/10.1038/s41467-01 8-07641-9.

75. Pritchard L, Glover RH, Humphris S, Elphinstone JG, Toth IK. Genomics and taxonomy in diagnostics for food security: soft-rotting enterobacterial plant pathogens. Analytical Methods. 2016;8(1):12-24. https://doi.org/10.1039/C5A $\mathrm{Y} 02550 \mathrm{H}$.

76. Chaumeil PA, Mussig AJ, Hugenholtz P, Parks DH. GTDB-Tk: a toolkit to classify genomes with the Genome Taxonomy Database. Bioinformatics. 2019. https://doi.org/10.1093/bioinformatics/btz848.

77. von Meijenfeldt FAB, Arkhipova K, Cambuy DD, Coutinho FH, Dutilh BE. Robust taxonomic classification of uncharted microbial sequences and bins with CAT and BAT. Genome Biol. 2019;20(1):217. https://doi.org/10.1186/s13 059-019-1817-x.

78. Stamatakis A, Aberer AJ, Goll C, Smith SA, Berger SA, Izquierdo-Carrasco F. RAxML-Light: a tool for computing terabyte phylogenies. Bioinformatics. 2012;28(15):2064-6. https://doi.org/10.1093/bioinformatics/bts309.

79. Ding W, Baumdicker F, Neher RA. panX: pan-genome analysis and exploration. Nucleic Acids Res. 2018;46:e5

80. Edgar RC. MUSCLE: a multiple sequence alignment method with reduced time and space complexity. BMC Bioinformatics. 2004;5(1):113. https://doi. org/10.1186/1471-2105-5-113.

81. Schloss PD, Westcott SL, Ryabin T, Hall JR, Hartmann M, Hollister EB, et al. Introducing mothur: open-source, platform-independent, community-supported software for describing and comparing microbial communities. Appl Environ Microbiol. 2009;75(23):7537-41. https://doi.org/10.1128/AEM.01541-09.

82. Griese M, Lange C, Soppa J. Ploidy in cyanobacteria. Fems Microbiol Lett 2011;323(2):124-31. https://doi.org/10.1111/j.1574-6968.2011.02368.x.

83. Bushnell B. BBMap: a fast, accurate, splice-aware aligner. Berkeley: Lawrence Berkeley National Lab (LBNL); 2014.

84. Menzel P, Ng KL, Krogh A. Fast and sensitive taxonomic classification for metagenomics with Kaiju. Nat Commun. 2016;7(1):11257. https://doi.org/1 0.1038 /ncomms 11257

85. Oksanen J, Blanchet FG, Friendly M, Kindt R, Legendre P, McGlinn D, et al. Vegan: Community Ecology Package. R Package version 2.5.61. 2019.

86. Anderson MJ. A new method for non-parametric multivariate analysis of variance. Austral Ecol. 2001;26:32-46.

87. Anderson MJ. Distance-based tests for homogeneity of multivariate dispersions. Biometrics. 2006;62(1):245-53. https://doi.org/10.1111/j.1541-042 0.2005.00440.x

88. Paradis E, Claude J, Strimmer K. APE: analyses of phylogenetics and evolution in R language. Bioinformatics. 2004;20(2):289-90. https://doi.org/1 0.1093/bioinformatics/btg412.

89. Bray JR, Curtis JT. An ordination of the upland forest communities of southern Wisconsin. Ecol Monogr. 1957;27(4):325-49. https://doi.org/10.23 07/1942268.

90. Legendre P, Anderson MJ. Distance-based redundancy analysis: testing multispecies responses in multifactorial ecological experiments. Ecol Monogr. 1999;69(1):1-24. https://doi.org/10.1890/0012-9615(1999)069[0001: DBRATM]2.0.CO;2.

91. McMurdie PJ, Holmes S. phyloseq: an R package for reproducible interactive analysis and graphics of microbiome census data. PLoS One. 2013;8:e61217.

92. Shepard RN. The analysis of proximities - multidimensional-scaling with an unknown distance function. Psychometrika. 1962;27(2):125-40. https://doi. org/10.1007/BF02289630.

93. Kruskal JB. Multidimensional-scaling by optimizing goodness of fit to a nonmetric hypothesis. Psychometrika. 1964;29(1):1-27. https://doi.org/10.1 007/BF02289565.

94. Harrell JF, Dupont C. Hmisc: harrell miscellaneous. R package version 4.1-1. https://CRAN.R-project.org/package=Hmisc.
95. Watts SC, Ritchie SC, Inouye M, Holt KE. FastSpar: rapid and scalable correlation estimation for compositional data. Bioinformatics. 2019;35(6): 1064-6. https://doi.org/10.1093/bioinformatics/bty734.

96. Friedman J, Alm EJ. Inferring correlation networks from genomic survey data. PLoS Comput Biol. 2012;8(9):e1002687. https://doi.org/10.1371/journal. pcbi. 1002687

97. Legendre P, Desdevises $Y$, Bazin E. A statistical test for host-parasite coevolution. Syst Biol. 2002;51(2):217-34. https://doi.org/10.1080/106351502 52899734.

98. Revell $\amalg$. Phytools: an R package for phylogenetic comparative biology (and other things). Methods Ecol Evol. 2012;3(2):217-23. https://doi.org/10.1111/ j.2041-210X.2011.00169.x

99. Huerta-Cepas J, Szklarczyk D, Heller D, Hernandez-Plaza A, Forslund SK, Cook H, et al. eggNOG 5.0: a hierarchical, functionally and phylogenetically annotated orthology resource based on 5090 organisms and 2502 viruses. Nucleic Acids Res. 2019;47(D1):D309-D14. https://doi.org/10.1093/nar/gky1085.

100. Parks DH, Tyson GW, Hugenholtz P, Beiko RG. STAMP: statistical analysis of taxonomic and functional profiles. Bioinformatics. 2014;30(21):3123-4. https://doi.org/10.1093/bioinformatics/btu494.

101. Boyd JA, Jungbluth SP, Leu AO, Evans PN, Woodcroft BJ, Chadwick GL, et al Divergent methyl-coenzyme $M$ reductase genes in a deep-subseafloor Archaeoglobi. ISME J. 2019;13(5):1269-79. https://doi.org/10.1038/s41396-01 8-0343-2.

102. Page AJ, Cummins CA, Hunt M, Wong VK, Reuter S, Holden MTG, et al. Roary: rapid large-scale prokaryote pan genome analysis. Bioinformatics. 2015;31(22):3691-3. https://doi.org/10.1093/bioinformatics/btv421.

103. Blin K, Wolf T, Chevrette MG, Lu X, Schwalen CJ, Kautsar SA, et al. antiSMASH 4.0-improvements in chemistry prediction and gene cluster boundary identification. Nucleic Acids Res. 2017;45(W1):W36-41. https://doi. org/10.1093/nar/gkx319

104. Navarro-Munoz JC, Selem-Mojica N, Mullowney MW, Kautsar SA, Tryon JH, Parkinson El, et al. A computational framework to explore large-scale biosynthetic diversity. Nat Chem Biol. 2020;16(1):60-8. https://doi.org/10.103 8/s41589-019-0400-9.

105. Machado D, Andrejev S, Tramontano M, Patil KR. Fast automated reconstruction of genome-scale metabolic models for microbial species and communities. Nucleic Acids Res. 2018;46(15):7542-53. https://doi.org/10.1 093/nar/gky537.

\section{Publisher's Note}

Springer Nature remains neutral with regard to jurisdictional claims in published maps and institutional affiliations.

Ready to submit your research? Choose BMC and benefit from:

- fast, convenient online submission

- thorough peer review by experienced researchers in your field

- rapid publication on acceptance

- support for research data, including large and complex data types

- gold Open Access which fosters wider collaboration and increased citations

- maximum visibility for your research: over $100 \mathrm{M}$ website views per year

At $\mathrm{BMC}$, research is always in progress.

Learn more biomedcentral.com/submissions 